\title{
An approximate Taylor method for Stochastic Functional Differential Equations via polynomial condition
}

\author{
Dušan D. Djordjević and Marija Milošević
}

\begin{abstract}
The subject of this paper is an analytic approximate method for a class of stochastic functional differential equations with coefficients that do not necessarily satisfy the Lipschitz condition nor linear growth condition but they satisfy some polynomial conditions. Also, equations from the observed class have unique solutions with bounded moments. Approximate equations are defined on partitions of the time interval and their drift and diffusion coefficients are Taylor approximations of the coefficients of the initial equation. Taylor approximations require Fréchet derivatives since the coefficients of the initial equation are functionals. The main results of this paper are the $L^{p}$ and almost sure convergence of the sequence of the approximate solutions to the exact solution of the initial equation. An example that illustrates the theoretical results and contains the proof of the existence, uniqueness and moment boundedness of the approximate solution is displayed.
\end{abstract}

\section{Introduction and preliminary results}

It is well known that most of the stochastic differential equations (SDEs) cannot be solved explicitly. Even more obvious is that the generalization of the SDEs, the stochastic functional differential equations (SFDEs) cannot

Key Words: Fréchet derivative; $L^{p}$ and almost sure convergence; Polynomial condition; Stochastic differential equations; Taylor approximation.

2010 Mathematics Subject Classification: Primary 60H10; Secondary 41A25, 41A58.

Received: 22.03.2021

Accepted: 30.04.2021 
be solved explicitly, although some sufficient conditions for the existence and uniqueness of the solution are satisfied. One analytic method to find their approximate solutions in an explicit form, or in a form suitable for the application of numerical methods, is going to be presented.

In many stochastic equations, it is not enough to predict the future solely on the present but, it is required to know some of the past if the argument is considered as the time. This way we naturally come across the SFDEs, especially, SDEs with constant delay, SDEs with time-dependent delay, periodic SFDEs, etc. There are lots of examples of these equations and some of them appear in various predator-prey and population growth models in biology, gene expression, and epidemiology models in medicine, in lots of models with an aftereffect in mechanics such as particle motion in liquids, controlled motion of rigid bodies, viscoelasticity and so on (see, for example, $[3,6,8,9,11,12,13,19,21,22,23])$. There are some numerical methods for SFDEs if the equation is not explicitly solvable, such as those from the papers $[15,17,24]$, among others. However, some problems do not require numerical methods but analytical ones since there may be some properties of the solutions of equations that should be examined, such as stability, asymptotics, moment boundedness, etc, (see [10], first of all). That is why sometimes it is easier to work with approximate solutions if the desired properties are inherited from the solutions of initial equations instead of some complicated numerical methods. This is why our analytical methods have an advantage over numerical methods.

Essentials of the analytic method considered in this paper go way back to Atalla [1,2]. Following Atalla's papers, Milošević, Jovanović and Janković constructed approximate solutions to various types of SDEs such as SFDEs [16], defined on a partition of the time interval. The coefficients of these equations are approximated by theirs Taylor series up to arbitrary derivatives and closeness of the exact and approximate solutions is measured in the sense of the $L^{p}$-norm and with probability one.

In most cases, the Lipschitz and linear growth conditions for the drift and diffusion coefficients are required, which guarantees the existence and uniqueness of a solution of an equation. This is what we want to avoid, especially since most of the coefficients of SDEs do not satisfy these conditions. Our idea is to weaken those conditions in a way that they can satisfy some other conditions instead (such as one-sided Lipschitz, generalized Khasminskiitype conditions, for example). The existence and uniqueness of the solution of the initial equation are assumed throughout the whole paper, but they can be proven if appropriate conditions are added. It was assumed in [16] that the derivatives of the following order than the biggest one used in Taylor approximation of the drift and diffusion coefficients are uniformly bounded and 
that both the drift and diffusion coefficients satisfy the linear growth condition. This time, only the boundedness in zero of these derivatives and the polynomial condition for both the drift and diffusion coefficients and their derivatives of these orders is assumed instead. This different approach considering a different class of functionals is our main motivation for this work.

Whole consideration in this paper is related to a complete filtered probability space $\left(\Omega, \mathcal{F},\left\{\mathcal{F}_{t}\right\}_{t \geq 0}, P\right)$ with the filtration $\left\{\mathcal{F}_{t}\right\}_{t \geq 0}$ which satisfies usual conditions, that is, it is non-decreasing, continuous from the right and $\mathcal{F}_{0}$ contains all $P$-null sets. Some necessary notations are going to be introduced. For every nonnegative $t$, let $w(t)=\left[w_{1}(t), \ldots, w_{d_{1}}(t)\right]^{T}, d_{1} \in \mathbb{N}$, be a standard $d_{1}$-dimensional Wiener process, $\mathcal{F}_{t}$-adapted and independent of $\mathcal{F}_{0}$. Let $|\cdot|_{E}$ or $|\cdot|_{F}$ represent the Euclidean norm or, more general, Frobenius (trace) norm of real vectors or matrices, that is, $|S|_{F}=\left(\operatorname{trace}\left(S^{T} S\right)\right)^{\frac{1}{2}}=\left(\sum_{i=1}^{k} \sum_{j=1}^{l} s_{i, j}^{2}\right)^{\frac{1}{2}}$, for $S=\left[s_{i, j}\right]_{k \times l}$. Notation $|\cdot|$ will also be used without further emphasizing, especially for normed space $\mathbb{R}$ where the Euclidean norm comes down to the absolute value. Additionally, $\langle\cdot, \cdot\rangle$ represents the standard Euclidean scalar product of vectors. Marks $\vee$ and $\wedge$ will represent maximum and minimum of real numbers, respectively.

Let $\tau, t_{0}$ and $T$, where $\tau>0$ and also $t_{0}<T$, be fixed real numbers and let us denote an important class of functions $V=\mathcal{C}\left([-\tau, 0], \mathbb{R}^{d}\right)$, where $V$ contains all continuous functions $\varphi:[-\tau, 0] \rightarrow \mathbb{R}^{d}$. It is familiar that $\mathbb{V}=\left(V,\|\cdot\|_{V}\right)$ is a Banach space, where $\|\cdot\|_{V}$ is the supremum norm, that is $\|\varphi\|_{V}=\sup _{[-\tau, 0]}|\varphi(\theta)|_{E}$ for every $\varphi \in V$.

Let us consider SFDE

$$
d x(t)=a\left(x_{t}, t\right) d t+b\left(x_{t}, t\right) d w(t), \quad t \in\left[t_{0}, T\right],
$$

with the given initial condition

$$
x_{t_{0}}=\eta=\{\eta(\theta) \mid \theta \in[-\tau, 0]\} .
$$

The appropriate integral form of Eq. (1) is

$$
x(t)=\eta(0)+\int_{t_{0}}^{t} a\left(x_{u}, u\right) d u+\int_{t_{0}}^{t} b\left(x_{u}, u\right) d w(u), \quad t \in\left[t_{0}, T\right],
$$

where $x_{t}$ represents the stochastic process $\{x(t+\theta) \mid \theta \in[-\tau, 0]\}$, that is $x_{t}(\theta)=x(t+\theta)$ for $\theta \in[-\tau, 0]$ and $t \in\left[t_{0}, T\right]$, and $x(t)$ is an $\mathbb{R}^{d}$-valued random variable for fixed $t \in\left[t_{0}-\tau, T\right]$. Also, $\eta$ is an $\mathcal{F}_{t_{0}}$-measurable, $V$ valued random variable. Mappings $a$ and $b$ from $V \times\left[t_{0}, T\right]$ to $\mathbb{R}^{d}$ and $\mathbb{R}^{d \times d_{1}}$, respectively, are Borel measurable.

For every positive, big enough integer $n$ let us consider an equidistant partition of the time interval $\left[t_{0}, T\right]$ of the form

$$
t_{0}<t_{1}<\cdots<t_{n}=T,
$$


that is, $t_{k}=t_{0}+k\left(T-t_{0}\right) / n, k \in\{0, \ldots, n\}$. The diameter of this partition is marked as $\delta_{n}=\left(T-t_{0}\right) / n<1$. On this partition, following approximate equations are going to be considered

$$
\begin{aligned}
x^{n}(t)= & x^{n}\left(t_{k}\right)+\int_{t_{k}}^{t} \sum_{i=0}^{m_{1}} \frac{a_{\left(x_{t_{k}}^{n}, s\right)}^{(i)}(\overbrace{x_{s}^{n}-x_{t_{k}}^{n}, \ldots, x_{s}^{n}-x_{t_{k}}^{n}}^{i \text { times }})}{i !} d s \\
& +\int_{t_{k}}^{t} \sum_{i=0}^{m_{2}} \frac{b_{\left(x_{t_{k}}^{n}, s\right)}^{(i)}(\overbrace{x_{s}^{n}-x_{t_{k}}^{n}, \ldots, x_{s}^{n}-x_{t_{k}}^{n}}^{i \text { times }})}{i !} d w(s), \quad t \in\left[t_{k}, t_{k+1}\right],
\end{aligned}
$$

whenever $k \in\{0,1, \ldots, n-1\}$, with initial condition (2) and initial conditions $x_{t_{k}}^{n}=\left\{x^{n}\left(t_{k}+\theta\right), \mid \theta \in[-\tau, 0]\right\}, k \in\{1, \ldots, n-1\}$, derived from the solutions of the previous equations. Solutions of Eqs. (5) are going to be used for approximation of the solution $x=\left\{x(t) \mid t \in\left[t_{0}-\tau, T\right]\right\}$ of Eq. (3) with initial condition (2). The approximate solution $x^{n}=\left\{x^{n}(t) \mid t \in\left[t_{0}-\tau, T\right]\right\}$, constructed by successive connecting of the initial condition (2) and processes $\left\{x^{n}(t) \mid t \in\left[t_{k}, t_{k+1}\right]\right\}$ in points $t_{k}, k \in\{0,1, \ldots, n-1\}$, is almost surely continuous process.

In Eqs. (5), the drift and diffusion coefficients are Taylor approximations of functionals $a$ and $b$, respectively. Here $a_{\left(x_{t_{k}}^{n}, s\right)}^{(i)}$ and $b_{\left(x_{t_{k}}^{n}, s\right)}^{(i)}$ represent $i$-th Fréchet derivatives of functionals $a$ and $b$ with respect to the first argument, respectively, in the points $\left(x_{t_{k}}^{n}, s\right)$, where $i \geq 1$. Thereby $a_{\left(x_{t_{k}}^{n}, s\right)}^{(0)}=a\left(x_{t_{k}}^{n}, s\right) \in$ $\mathbb{R}^{d}$ and $b_{\left(x_{t_{k}}^{n}, s\right)}^{(0)}=b\left(x_{t_{k}}^{n}, s\right) \in \mathbb{R}^{d \times d_{1}}$.

In the sequel, we introduce the basic notion of the Fréchet derivative (see, for example, $[4,5])$. Let $\mathbb{X}=\left(X,\|\cdot\|_{1}\right)$ and $\mathbb{Y}=\left(Y,\|\cdot\|_{2}\right)$ be normed spaces over the same field $\mathbb{F}$ and $\mathcal{L}(X, Y)$ represents the space of all bounded (continuous) linear operators from $X$ to $Y$. The norm $\|\cdot\|_{\mathcal{L}(X, Y)}$ in $\mathcal{L}(X, Y)$ is defined as $\|A\|_{\mathcal{L}(X, Y)}=\sup _{\|x\|_{1} \leq 1}\|A x\|_{2}$ for $A \in \mathcal{L}(X, Y)$ and $\left(\mathcal{L}(X, Y),\|\cdot\|_{\mathcal{L}(X, Y)}\right)$ is a Banach space over the same field $\mathbb{F}$. Let $U$ be an open subset of $X$, functional $f: U \rightarrow Y$ and $x_{0} \in U$. If there exists some $F \in \mathcal{L}(X, Y)$ such that

$$
\lim _{\|h\|_{1} \rightarrow 0} \frac{\left\|f\left(x_{0}+h\right)-f\left(x_{0}\right)-F h\right\|_{2}}{\|h\|_{1}}=0,
$$

then $F$ is the Fréchet derivative of $f$ at $x_{0}$ and the notation used here is $F=f_{x_{0}}^{\prime}$. Previous limit is taken as the vector $h$ tends to zero in $X$ and the Fréchet derivative is unique in the case when it exists.

Let $f: U \rightarrow Y$ be a mapping which is Fréchet differentiable at every point $x_{0} \in U$. In this case, $f_{x_{0}}^{\prime} \in \mathcal{L}(X, Y)$. We can consider the mapping $u \mapsto f_{u}^{\prime}$ 
from $U$ to $\mathcal{L}(X, Y)$. If this mapping is Fréchet differentiable at $x_{0}$, then the second Fréchet derivative is $f_{x_{0}}^{\prime \prime} \in \mathcal{L}(X, \mathcal{L}(X, Y))$. In the case when the second Fréchet derivative exists in some neighborhood $U$ of the vector $x_{0} \in X$, we can define $F: U \rightarrow \mathcal{L}(X, Y)$ as $F(x)=f_{x}^{\prime}$, for every $x \in U$. Then, we have

$$
\begin{aligned}
\lim _{\|h\|_{1} \rightarrow 0} & \frac{\left\|F\left(x_{0}+h\right)-F\left(x_{0}\right)-F_{x_{0}}^{\prime} h\right\|_{\mathcal{L}(X, Y)}}{\|h\|_{1}} \\
& =\lim _{\|h\|_{1} \rightarrow 0} \frac{\left\|f_{x_{0}+h}^{\prime}-f_{x_{0}}^{\prime}-f_{x_{0}}^{\prime \prime} h\right\|_{\mathcal{L}(X, Y)}}{\|h\|_{1}}=0 .
\end{aligned}
$$

Let $\mathbb{X}_{1}=\left(X_{1},\|\cdot\|_{1}\right), \ldots, \mathbb{X}_{n}=\left(X_{n},\|\cdot\|_{n}\right), \mathbb{Y}=\left(Y,\|\cdot\|_{Y}\right)$ be normed spaces over the same field $\mathbb{F}$ and let $B: X_{1} \times \cdots \times X_{n} \rightarrow Y$ be a mapping, which is linear in every argument. Then $B$ is an $n$-linear operator from $X_{1} \times \cdots \times X_{n}$ to $Y$ and $B$ is bounded if there exists some constant $M \geq 0$ such that, for all $\left(x_{1}, \ldots, x_{n}\right) \in X_{1} \times \cdots \times X_{n}$, the following holds

$$
\left\|B\left(x_{1}, \ldots, x_{n}\right)\right\|_{Y} \leq M\left\|x_{1}\right\|_{1} \cdots\left\|x_{n}\right\|_{n} .
$$

The set of all bounded $n$-linear operators from $X_{1} \times \cdots \times X_{n}$ to $Y$ is denoted by $\mathcal{T}\left(X_{1}, \ldots, X_{n} ; Y\right)$. Shortly, $\mathcal{T}(X, \ldots, X ; Y) \equiv \mathcal{T}\left(X^{n} ; Y\right)$ and $\mathcal{T}\left(X_{1}, \ldots, X_{n} ; Y\right)$ is a vector space. Moreover, if we define the norm of $B \in \mathcal{T}\left(X_{1}, \ldots, X_{n} ; Y\right)$ as the infimum of all admissible $M$ in the inequality (6), then $\mathcal{T}\left(X_{1}, \ldots, X_{n} ; Y\right)$ is a normed space. The norm of $B$, obtained in a described way, is denoted by $\|B\|_{M, n}$. This norm is equivalently defined as

$$
\|B\|_{M, n}=\sup _{\substack{h_{1} \in X_{1}, \ldots, h_{n} \in X_{n} \\\left\|h_{1}\right\|_{1}=\cdots=\left\|h_{n}\right\|_{n}=1}}\left\|B\left(h_{1}, \ldots, h_{n}\right)\right\|_{Y}=\inf \{M \geq 0 \mid M \text { satisfies (6) }\} .
$$

Multi-linear and bounded linear operators are in a close relation in a sense that $\mathcal{T}\left(X^{n} ; Y\right)$ is isometrically isomorphic to $\left.\left.\mathcal{L}(\underbrace{X, \mathcal{L}(X, \ldots, \mathcal{L}(X}_{n \text { times }}, Y) \ldots\right)\right)$ with respect to standard norms on these spaces. So, if $x \in X$, then $f_{x_{0}}^{\prime \prime}(x) \in$ $\mathcal{L}(X, Y)$. If $y \in X$ as well, then $f_{x_{0}}^{\prime \prime}(x)(y)=f_{x_{0}}^{\prime \prime}(x, y)$ belongs to $Y$ and the mapping $(x, y) \mapsto f_{x_{0}}^{\prime \prime}(x, y)$ belongs to $\mathcal{T}\left(X^{2} ; Y\right)$. The norm $\left\|f_{x_{0}}^{\prime \prime}\right\|_{\mathcal{L}(X, \mathcal{L}(X, Y))}$ $=\left\|f_{x_{0}}^{\prime \prime}\right\|_{M, 2}$ is the same in the space $\mathcal{L}(X, \mathcal{L}(X, Y))$ and in the space $\mathcal{T}\left(X^{2} ; Y\right)$.

In the same manner higher Fréchet derivatives can be defined, in the case when they do exist. Thus, the $n$-th Fréchet derivative of the function $f$ at $x_{0}$ is

$$
\left.\left.f_{x_{0}}^{(n)} \in \mathcal{L}(\underbrace{X, \mathcal{L}(X, \ldots, \mathcal{L}(X}_{n \text { times }}, Y) \ldots\right)\right)
$$

if the function $x_{0} \mapsto f_{x_{0}}^{(n-1)}$ is Fréchet differentiable in some neighborhood of $x_{0}$. 
Let us recall the Taylor formula $[4,5]$. Let $\mathbb{X}=\left(X,\|\cdot\|_{1}\right)$ and $\mathbb{Y}=\left(Y,\|\cdot\|_{2}\right)$ be normed spaces over the same field $\mathbb{F}$, let $U$ be an open subset of $X$ and let $f: U \rightarrow Y$ be $(n+1)$-times Fréchet differentiable. Assume that $x_{0}, x \in U$, such that the segment $\left[x_{0}, x\right] \subset U$ (that is, $x_{0}+\theta\left(x-x_{0}\right) \in U$ for every $0 \leq \theta \leq 1)$. Then the following formula holds

$$
\begin{aligned}
f(x)-f\left(x_{0}\right)= & \sum_{k=1}^{n} \frac{1}{k !} f_{x_{0}}^{(k)}(\underbrace{x-x_{0}, \ldots, x-x_{0}}_{k \text { times }}) \\
& +\frac{1}{(n+1) !} f_{x_{0}+\bar{\theta}\left(x-x_{0}\right)}^{(n+1)}(\underbrace{x-x_{0}, \ldots, x-x_{0}}_{n+1 \text { times }}),
\end{aligned}
$$

for some $\bar{\theta} \in(0,1)$. Notice that the $k$-th Fréchet derivative is a $k$-linear operator, so the notation $f_{x_{0}}^{(k)}(\underbrace{x-x_{0}, \ldots, x-x_{0}}_{k \text { times }}) \equiv f_{x_{0}}^{(k)}\left(x-x_{0}\right)^{k}$ may also be used throughout the paper. The residuum of the Taylor approximation can be estimated as

$$
\begin{aligned}
\left|R\left(x_{0}, h\right)\right|_{E} & \equiv \frac{1}{(n+1) !}\left|f_{x_{0}+\bar{\theta} h}^{(n+1)}(\underbrace{h, \ldots, h}_{n+1 \text { times }})\right|_{E} \\
& \leq \frac{1}{(n+1) !} \sup _{\theta \in[0,1]}\left\|f_{x_{0}+\theta h}^{(n+1)}\right\|_{M, n+1}\|h\|^{n+1},
\end{aligned}
$$

for $h=x-x_{0} \in X$.

For easier managing in future analysis, the following assumptions are categorically listed.

$\mathcal{A}_{1}$ : There exist Taylor expansions of the functionals $a$ and $b$ in the first argument up to the $m_{1}$-th and $m_{2}$-th Fréchet derivatives, respectively.

$\mathcal{A}_{2}$ : The initial data $\eta$ satisfies the polynomial condition, that is, there exist positive constant $D^{\prime}$ and a nonnegative integer $q^{\prime}$ such that, for every $\theta_{1}, \theta_{2} \in[-\tau, 0]$,

$$
\left|\eta\left(\theta_{1}\right)-\eta\left(\theta_{2}\right)\right|_{E}^{2} \leq D^{\prime}\left(1+\left|\theta_{1}\right|^{q^{\prime}}+\left|\theta_{2}\right|^{q^{\prime}}\right)\left|\theta_{1}-\theta_{2}\right|^{2} .
$$

$\mathcal{A}_{3}$ : Functions $a(0, \cdot)$ and $b(0, \cdot)$ are bounded on $\left[t_{0}, T\right]$, that is, there exist positive constants $K_{a}$ and $K_{b}$, such that $|a(0, t)|_{E} \leq K_{a}$ and $|b(0, t)|_{E} \leq K_{b}$, for every $t_{0} \leq t \leq T$.

$\mathcal{A}_{4}$ : Functions $a_{(0, \cdot)}^{\left(m_{1}+1\right)}$ and $b_{(0, \cdot)}^{\left(m_{2}+1\right)}$ are bounded on $\left[t_{0}, T\right]$, that is, there exist positive constants $K_{a}^{\prime}$ and $K_{b}^{\prime}$ such that, for every $t \in\left[t_{0}, T\right]$,

$$
\left\|a_{(0, t)}^{\left(m_{1}+1\right)}\right\|_{M, m_{1}+1} \leq K_{a}^{\prime}, \quad\left\|b_{(0, t)}^{\left(m_{2}+1\right)}\right\|_{M, m_{2}+1} \leq K_{b}^{\prime} .
$$


$\mathcal{A}_{5}$ : The functionals $a, b$ and theirs Fréchet derivatives with respect to the first argument of order $m_{1}+1$ and $m_{2}+1$, respectively, satisfy following polynomial conditions: there exist positive real numbers $D_{a}, D_{b}, D_{a}^{\prime}$ and $D_{b}^{\prime}$ and there exist nonnegative integers $q_{a}, q_{b}, q_{a}^{\prime}$ and $q_{b}^{\prime}$ such that, for every $x, y \in V$ and $t \in\left[t_{0}, T\right]$,

$$
\begin{aligned}
& |a(x, t)-a(y, t)|_{E}^{2} \leq D_{a}\left(1+\|x\|_{V}^{q_{a}}+\|y\|_{V}^{q_{a}}\right)\|x-y\|_{V}^{2}, \\
& |b(x, t)-b(y, t)|_{E}^{2} \leq D_{b}\left(1+\|x\|_{V}^{q_{b}}+\|y\|_{V}^{q_{b}}\right)\|x-y\|_{V}^{2}, \\
& \left\|a_{(x, t)}^{\left(m_{1}+1\right)}-a_{(y, t)}^{\left(m_{1}+1\right)}\right\|_{M, m_{1}+1}^{2} \leq D_{a}^{\prime}\left(1+\|x\|_{V}^{q_{a}^{\prime}}+\|y\|_{V}^{q_{a}^{\prime}}\right)\|x-y\|_{V}^{2}, \\
& \left\|b_{(x, t)}^{\left(m_{2}+1\right)}-b_{(y, t)}^{\left(m_{2}+1\right)}\right\|_{M, m_{2}+1}^{2} \leq D_{b}^{\prime}\left(1+\|x\|_{V}^{q_{b}^{\prime}}+\|y\|_{V}^{q_{b}^{\prime}}\right)\|x-y\|_{V}^{2} .
\end{aligned}
$$

$\mathcal{A}_{6}$ : There exist unique solutions $x$ and $x^{n}$ of Eqs. (3) and (5), respectively, such that for $p \geq 2$,

$$
\begin{aligned}
& E \sup _{t \in\left[t_{0}-\tau, T\right]}|x(t)|_{E}^{2 p(1 \vee q)} \leq Q<\infty, \\
& E \sup _{t \in\left[t_{0}-\tau, T\right]}\left|x^{n}(t)\right|_{E}^{2 p[1+(M \vee(q / 2))][2+M+(M \vee(q / 2))]} \leq Q<\infty,
\end{aligned}
$$

where $Q$ is a positive constant, independent of $n, q=q_{a} \vee q_{b} \vee q_{a}^{\prime} \vee q_{b}^{\prime}$ and $M=m_{1} \vee m_{2}$.

The existence and uniqueness of solutions of Eqs. (3) and (5) are assumed without considering any conditions which are satisfied by their coefficients. However, the example will illustrate that the class of SFDEs which satisfy the existence and uniqueness conditions, as well as the assumptions $\mathcal{A}_{1}-\mathcal{A}_{6}$ is not empty. All Lebesgue and Itô integrals are supposed to be well defined.

In this paper, some known inequalities, such as Hölder and BurkholderDavis-Gundy inequality are used in subsequent proofs. Likewise, the elementary inequality is used in the sequel: for every $r \geq 0$, normed space $\left(N,|\cdot|_{N}\right)$ and $a_{i} \in N, i \in\{1,2, \ldots, n\}, n \in \mathbb{N}$,

$$
\left|\sum_{i=1}^{n} a_{i}\right|_{N}^{r} \leq\left(n^{r-1} \vee 1\right) \cdot \sum_{i=1}^{n}\left|a_{i}\right|_{N}^{r} .
$$

Following theorem plays an important part in the further analysis and it represents one of the integral Bihari-type inequalities from [18].

Theorem 1.1. Let $\mathcal{G}$ be the class of functions $\varphi: \mathbb{R}^{+} \rightarrow \mathbb{R}^{+}$which satisfy the following conditions:

1) $\varphi$ is nondecreasing and continuous in $\mathbb{R}^{+}$and $\varphi(u)>0$ for $u>0$;

2) $\frac{1}{\alpha} \varphi(u) \leq \varphi\left(\frac{u}{\alpha}\right), u \geq 0, \alpha \geq 1$. 
Let $f=f(x), u=u(x)$ be a real-valued nonnegative continuous functions on $U$, where $U$ is any bounded open set in $\mathbb{R}$. If $g=g(x)$ is a positive, nondecreasing continuous function on $U$ and $\varphi$ belongs to the class $\mathcal{G}$ for which the following inequality

$$
u(x) \leq g(x)+\int_{x^{0}}^{x} f(t) \varphi(u(t)) d t
$$

holds for all $x \in U$ with $x \geq x^{0} \in U$, then for $x^{0} \leq x \leq x^{*}$,

$$
u(x) \leq g(x) G^{-1}\left(G(1)+\int_{x^{0}}^{x} f(t) d t\right),
$$

where

$$
G(z)=\int_{z^{0}}^{z} \frac{d s}{\varphi(s)}, z \geq z^{0}>0
$$

and $G^{-1}$ is the inverse function of $G$, where $x^{*}$ is chosen so that

$$
G(1)+\int_{x^{0}}^{x} f(t) d t \in \operatorname{Dom}\left(G^{-1}\right) .
$$

\section{Main results}

Our main goal in this section and in whole paper is to prove the $L^{p}$ and almost sure convergence of the processes $x^{n}=\left\{x^{n}(s) \mid s \in\left[t_{0}-\tau, T\right]\right\}$, which are the solutions of Eqs. (5), to the process $x=\left\{x(s) \mid s \in\left[t_{0}-\tau, T\right]\right\}$ which is the solution to Eq. (3) with initial condition (2). In order to do that, we established two necessary propositions.

Proposition 1. Let $\left\{x^{n}(t) \mid t \in\left[t_{k}-\tau, t_{k+1}\right]\right\}, k \in\{0, \ldots, n-1\}$ represent the solutions of Eqs. (5) with the initial condition (2) and let us assume that the conditions $\mathcal{A}_{1}, \mathcal{A}_{3}-\mathcal{A}_{6}$ are satisfied. Then, for every $0<r \leq(M+2+$ $\left.\left[M \vee \frac{q}{2}\right]\right) p$,

$$
E \sup _{s \in\left[t_{k}, t\right]}\left|x^{n}(s)-x^{n}\left(t_{k}\right)\right|_{E}^{r} \leq C \delta_{n}^{r / 2}, \quad t \in\left[t_{k}, t_{k+1}\right], k \in\{0, \ldots, n-1\},
$$

where $C$ is a generic constant independent of both $n$ and $\delta_{n}$.

Proof. Let us introduce

$$
\begin{aligned}
A\left(x_{t}^{n}, t ; x_{t_{k}}^{n}\right) & =\sum_{i=0}^{m_{1}} \frac{a_{\left(x_{t_{k}}^{n}, t\right)}^{(i)}\left(x_{t}^{n}-x_{t_{k}}^{n}, \ldots, x_{t}^{n}-x_{t_{k}}^{n}\right)}{i !}, \\
B\left(x_{t}^{n}, t ; x_{t_{k}}^{n}\right) & =\sum_{i=0}^{m_{2}} \frac{b_{\left(x_{t_{k}}^{n}, t\right)}^{(i)}\left(x_{t}^{n}-x_{t_{k}}^{n}, \ldots, x_{t}^{n}-x_{t_{k}}^{n}\right)}{i !}
\end{aligned}
$$


for simplicity.

Afterward, only the case where $r \geq 2$ is considered. The case $0<r<2$ can be then easily proved by using the Hölder inequality with the conjugate exponents $(2 / r, 2 /(2-r))$.

Using the inequality (8), Hölder inequality for the appropriate Lebesgue integral with the conjugate exponents $(r, r /(r-1))$, Burkholder-Davis-Gundy and Hölder inequality with the conjugate exponents $(r / 2, r /(r-2))$ (for $r>2)$, Itô isometry, Doob martingale inequality (for $r=2$ ) and Fubini theorem for the appropriate stochastic integral, it is easy to obtain

$$
E \sup _{s \in\left[t_{k}, t\right]}\left|x^{n}(s)-x^{n}\left(t_{k}\right)\right|_{E}^{r} \leq 2^{r-1}\left(t-t_{k}\right)^{\frac{r}{2}-1}\left[\left(t-t_{k}\right)^{r / 2} J_{1}(t)+c_{r} J_{2}(t)\right]
$$

where

$$
J_{1}(t)=\int_{t_{k}}^{t} E\left|A\left(x_{s}^{n}, s ; x_{t_{k}}^{n}\right)\right|_{E}^{r} d s, \quad J_{2}(t)=\int_{t_{k}}^{t} E\left|B\left(x_{s}^{n}, s ; x_{t_{k}}^{n}\right)\right|_{E}^{r} d s
$$

and the constant $c_{r}>0$ is obtained from the Burkholder-Davis-Gundy inequality.

Let us now estimate $J_{1}(t)$ using the assumption $\mathcal{A}_{1}$ and inequality (8). For simplicity, let us introduce a new mark $\Delta_{s}^{n}=x_{s}^{n}-x_{t_{k}}^{n}$, for $s \in\left[t_{k}, t_{k+1}\right]$, $k \in\{0,1, \ldots, n-1\}$. There exists $\bar{\theta} \in(0,1)$ such that

$$
\begin{aligned}
& J_{1}(t) \\
& =\int_{t_{k}}^{t} E\left|a\left(x_{s}^{n}, s\right)-\left[a\left(x_{s}^{n}, s\right)+A\left(x_{s}^{n}, s ; x_{t_{k}}^{n}\right)\right]\right|_{E}^{r} d s \\
& =\int_{t_{k}}^{t} E\left|a\left(x_{s}^{n}, s\right)-\frac{a_{\left(x_{t_{k}}^{n}+\bar{\theta} \Delta_{s}^{n}, s\right)}^{\left(m_{1}+1\right)}\left(\Delta_{s}^{n}, \ldots, \Delta_{s}^{n}\right)}{\left(m_{1}+1\right) !}\right|_{E}^{r} d s \\
& \leq 2^{r-1}\left[\int_{t_{k}}^{t} E\left|a\left(x_{s}^{n}, s\right)\right|_{E}^{r} d s+\frac{1}{\left[\left(m_{1}+1\right) !\right]^{r}} \int_{t_{k}}^{t} E\left|a_{\left(x_{t_{k}}^{n}+\bar{\theta} \Delta_{s}^{n}, s\right)}^{\left(m_{1}+1\right)}\left(\Delta_{s}^{n}, \ldots, \Delta_{s}^{n}\right)\right|_{E}^{r} d s\right] .
\end{aligned}
$$

Following part will be proved using the relation (8) and $\mathcal{A}_{3}, \mathcal{A}_{5}$ and $\mathcal{A}_{6}$, such that

$$
\begin{aligned}
\int_{t_{k}}^{t} E \mid & \left.a\left(x_{s}^{n}, s\right)\right|_{E} ^{r} d s \\
& =\int_{t_{k}}^{t} E\left|a\left(x_{s}^{n}, s\right)-a(0, s)+a(0, s)\right|_{E}^{r} d s \\
& \leq 2^{r-1}\left[\int_{t_{k}}^{t} E\left(\left|a\left(x_{s}^{n}, s\right)-a(0, s)\right|_{E}^{2}\right)^{r / 2} d s+K_{a}^{r}\left(t-t_{k}\right)\right]
\end{aligned}
$$




$$
\begin{aligned}
& \leq 2^{r-1}\left[D_{a}^{r / 2} \int_{t_{k}}^{t} E\left(1+\left\|x_{s}^{n}\right\|_{V}^{q_{a}}\right)^{r / 2}\left\|x_{s}^{n}\right\|_{V}^{r} d s+K_{a}^{r}\left(t-t_{k}\right)\right] \\
& \leq 2^{r-1}\left[D_{a}^{r / 2} 2^{r / 2-1}\left(\int_{t_{k}}^{t} E\left\|x_{s}^{n}\right\|_{V}^{r} d s+\int_{t_{k}}^{t} E\left\|x_{s}^{n}\right\|_{V}^{r\left(1+q_{a} / 2\right)} d s\right)+K_{a}^{r}\left(t-t_{k}\right)\right] \\
& \leq 2^{r-1}\left[D_{a}^{r / 2} 2^{r / 2}(1+Q)\left(t-t_{k}\right)+K_{a}^{r}\left(t-t_{k}\right)\right]=K^{\prime}\left(t-t_{k}\right)
\end{aligned}
$$

where

$$
K^{\prime}=2^{r-1}\left(D_{a}^{r / 2} 2^{r / 2}(1+Q)+K_{a}^{r}\right)
$$

is a constant independent of $n$ and $\delta_{n}$.

By applying (7), the assumptions $\mathcal{A}_{4}-\mathcal{A}_{6}$ as well as (8), we get

$$
\begin{aligned}
& \left|a_{\left(x_{t_{k}}^{n}+\bar{\theta} \Delta_{s}^{n}, s\right)}^{\left(m_{1}+1\right)}\left(\Delta_{s}^{n}, \ldots, \Delta_{s}^{n}\right)\right|_{E}^{r} \\
& \leq\left\|a_{\left(x_{t_{k}}^{n}+\bar{\theta} \Delta_{s}^{n}, s\right)}^{\left(m_{1}+1\right)}-a_{(0, s)}^{\left(m_{1}+1\right)}+a_{(0, s)}^{\left(m_{1}+1\right)}\right\|_{M, m_{1}+1}^{r} \cdot\left\|\Delta_{s}^{n}\right\|_{V}^{r\left(m_{1}+1\right)} \\
& \leq 2^{r-1}\left[\left(D_{a}^{\prime}\left(1+\left\|x_{t_{k}}^{n}+\bar{\theta} \Delta_{s}^{n}\right\|_{V}^{q_{a}^{\prime}}\right)\left\|x_{t_{k}}^{n}+\bar{\theta} \Delta_{s}^{n}\right\|_{V}^{2}\right)^{r / 2}+\left(K_{a}^{\prime}\right)^{r}\right]\left\|\Delta_{s}^{n}\right\|_{V}^{r\left(m_{1}+1\right)} \\
& \leq 2^{3 r / 2-2}\left(D_{a}^{\prime}\right)^{r / 2}\left[\left(\left\|x_{t_{k}}^{n}+\bar{\theta} \Delta_{s}^{n}\right\|_{V}^{r}+\left\|x_{t_{k}}^{n}+\bar{\theta} \Delta_{s}^{n}\right\|_{V}^{r\left(1+q_{a}^{\prime} / 2\right)}\right)\left\|\Delta_{s}^{n}\right\|_{V}^{r\left(m_{1}+1\right)}\right] \\
& \quad+2^{r-1}\left(K_{a}^{\prime}\right)^{r}\left\|\Delta_{s}^{n}\right\|_{V}^{r\left(m_{1}+1\right)} .
\end{aligned}
$$

The second summand on the right-hand side of (11) can now be estimated based on (13) via the inequality (8). Thus,

$$
\begin{aligned}
& \int_{t_{k}}^{t} E\left|a_{\left(x_{t_{k}}^{n}+\bar{\theta} \Delta_{s}^{n}, s\right)}^{\left(m_{1}+1\right)}\left(\Delta_{s}^{n}, \ldots, \Delta_{s}^{n}\right)\right|_{E}^{r} d s \\
& \leq 2^{3 r / 2-2}\left(D_{a}^{\prime}\right)^{r / 2}\left[\int_{t_{k}}^{t} E\left\|x_{t_{k}}^{n}+\bar{\theta} \Delta_{s}^{n}\right\|_{V}^{r}\left\|\Delta_{s}^{n}\right\|_{V}^{r\left(m_{1}+1\right)} d s\right. \\
& \left.\quad+\int_{t_{k}}^{t} E\left\|x_{t_{k}}^{n}+\bar{\theta} \Delta_{s}^{n}\right\|_{V}^{r\left(1+q_{a}^{\prime} / 2\right)}\left\|\Delta_{s}^{n}\right\|_{V}^{r\left(m_{1}+1\right)} d s\right] \\
& \quad+2^{r-1}\left(K_{a}^{\prime}\right)^{r} \int_{t_{k}}^{t} E\left\|\Delta_{s}^{n}\right\|_{V}^{r\left(m_{1}+1\right)} d s \\
& \leq\left(D_{a}^{\prime}\right)^{r / 2} 2^{r\left(m_{1}+7 / 2\right)-4} \\
& \quad\left\{\int_{t_{k}}^{t} E\left(|1-\bar{\theta}|^{r}\left\|x_{t_{k}}^{n}\right\|_{V}^{r}+|\bar{\theta}|^{r}\left\|x_{s}^{n}\right\|_{V}^{r}\right)\left(\left\|x_{s}^{n}\right\|_{V}^{r\left(m_{1}+1\right)}+\left\|x_{t_{k}}^{n}\right\|_{V}^{r\left(m_{1}+1\right)}\right) d s\right.
\end{aligned}
$$


AN APPROXIMATE TAYLOR METHOD FOR STOCHASTIC FUNCTIONAL DIFFERENTIAL EQUATIONS VIA POLYNOMIAL CONDITIONS

$$
\begin{gathered}
+2^{r q_{a}^{\prime} / 2 \int_{t_{k}}^{t} E}\left[\left(|1-\bar{\theta}|^{r\left(1+q_{a}^{\prime} / 2\right)}\left\|x_{t_{k}}^{n}\right\|_{V}^{r\left(1+q_{a}^{\prime} / 2\right)}+|\bar{\theta}|^{r\left(1+q_{a}^{\prime} / 2\right)}\left\|x_{s}^{n}\right\|_{V}^{r\left(1+q_{a}^{\prime} / 2\right)}\right)\right. \\
\left.\left.\cdot\left(\left\|x_{s}^{n}\right\|_{V}^{r\left(m_{1}+1\right)}+\left\|x_{t_{k}}^{n}\right\|_{V}^{r\left(m_{1}+1\right)}\right)\right] d s\right\} \\
+\left(K_{a}^{\prime}\right)^{r} 2^{r\left(m_{1}+2\right)-2} \int_{t_{k}}^{t} E\left(\left\|x_{s}^{n}\right\|_{V}^{r\left(m_{1}+1\right)}+\left\|x_{t_{k}}^{n}\right\|_{V}^{r\left(m_{1}+1\right)}\right) d s .
\end{gathered}
$$

Applying the Hölder inequality with the conjugate exponents $(2,2)$ for the terms of the form $E\left(\left\|x_{s}^{n}\right\|_{V}^{\alpha}\left\|x_{t_{k}}^{n}\right\|_{V}^{\beta}\right)$ in the previous estimates, that is, the Cauchy-Schwarz-Bunyakovsky inequality, recalling that $0<\theta<1$ and finally applying the assumption $\mathcal{A}_{6}$, we can attain

$$
\begin{aligned}
& \int_{t_{k}}^{t} E\left|a_{\left(x_{t_{k}}^{n}+\bar{\theta} \Delta_{s}^{n}, s\right)}^{\left(m_{1}+1\right)}\left(\Delta_{s}^{n}, \ldots, \Delta_{s}^{n}\right)\right|_{E}^{r} d s \\
& \leq 2^{r\left(m_{1}+7 / 2\right)-4}\left(D_{a}^{\prime}\right)^{r / 2} \\
& \left\{\left[(1-\bar{\theta})^{r}\left(\int_{t_{k}}^{t}\left(E\left\|x_{t_{k}}^{n}\right\|_{V}^{2 r}\right)^{1 / 2}\left(E\left\|x_{s}^{n}\right\|_{V}^{2 r\left(m_{1}+1\right)}\right)^{1 / 2} d s+\int_{t_{k}}^{t} E\left\|x_{t_{k}}^{n}\right\|_{V}^{r\left(m_{1}+2\right)} d s\right)\right.\right. \\
& \left.+\bar{\theta}^{r}\left(\int_{t_{k}}^{t} E\left\|x_{s}^{n}\right\|_{V}^{r\left(m_{1}+2\right)} d s+\int_{t_{k}}^{t}\left(E\left\|x_{s}^{n}\right\|_{V}^{2 r}\right)^{1 / 2}\left(E\left\|x_{t_{k}}^{n}\right\|_{V}^{2 r\left(m_{1}+1\right)}\right)^{1 / 2} d s\right)\right] \\
& +2^{r q_{a}^{\prime} / 2}\left[( 1 - \overline { \theta } ) ^ { r ( 1 + q _ { a } ^ { \prime } / 2 ) } \left(\int_{t_{k}}^{t}\left(E\left\|x_{t_{k}}^{n}\right\|_{V}^{2 r\left(1+q_{a}^{\prime} / 2\right)}\right)^{1 / 2}\left(E\left\|x_{s}^{n}\right\|_{V}^{2 r\left(m_{1}+1\right)}\right)^{1 / 2} d s\right.\right. \\
& \left.+\int_{t_{k}}^{t} E\left\|x_{t_{k}}^{n}\right\|_{V}^{r\left(m_{1}+2+q_{a}^{\prime} / 2\right)} d s\right) \\
& +\bar{\theta}^{r\left(1+q_{a}^{\prime} / 2\right)}\left(\int_{t_{k}}^{t} E\left\|x_{s}^{n}\right\|_{V}^{r\left(m_{1}+2+q_{a}^{\prime} / 2\right)} d s\right. \\
& \left.\left.\left.+\int_{t_{k}}^{t}\left(E\left\|x_{s}^{n}\right\|_{V}^{2 r\left(1+q_{a}^{\prime} / 2\right)}\right)^{1 / 2}\left(E\left\|x_{t_{k}}^{n}\right\|_{V}^{2 r\left(m_{1}+1\right)}\right)^{1 / 2} d s\right)\right]\right\} \\
& +2^{r\left(m_{1}+2\right)-2}\left(K_{a}^{\prime}\right)^{r} \int_{t_{k}}^{t}\left(E\left\|x_{s}^{n}\right\|_{V}^{r\left(m_{1}+1\right)}+E\left\|x_{t_{k}}^{n}\right\|_{V}^{r\left(m_{1}+1\right)}\right) d s \\
& \leq 2^{r\left(m_{1}+2\right)-1}(1+Q)\left(t-t_{k}\right) \\
& \cdot\left\{\left(K_{a}^{\prime}\right)^{r}+\left(D_{a}^{\prime}\right)^{r / 2} 2^{3 r / 2-2}\left[\left((1-\bar{\theta})^{r}+\bar{\theta}^{r}\right)+2^{r q_{a}^{\prime} / 2}\left((1-\bar{\theta})^{r\left(1+q_{a}^{\prime} / 2\right)}+\bar{\theta}^{r\left(1+q_{a}^{\prime} / 2\right)}\right)\right]\right\} \\
& \leq K^{\prime \prime}\left(t-t_{k}\right)
\end{aligned}
$$


where

$$
K^{\prime \prime}=2^{r\left(m_{1}+2\right)-1}(1+Q)\left[\left(D_{a}^{\prime}\right)^{r / 2} 2^{3 r / 2-1}\left(1+2^{r q_{a}^{\prime} / 2}\right)+\left(K_{a}^{\prime}\right)^{r}\right] .
$$

Putting (12) and (14) into (11), we compute

$$
J_{1}(t) \leq 2^{r-1}\left[K^{\prime}+\left[\left(m_{1}+1\right) !\right]^{-r} K^{\prime \prime}\right]\left(t-t_{k}\right) .
$$

Analogous procedure for $J_{2}(t)$ gives

$$
J_{2}(t) \leq 2^{r-1}\left[L^{\prime}+\left[\left(m_{2}+1\right) !\right]^{-r} L^{\prime \prime}\right]\left(t-t_{k}\right),
$$

where

$$
\begin{aligned}
& L^{\prime}=2^{r-1}\left(D_{b}^{r / 2} 2^{r / 2}(1+Q)+K_{b}^{r}\right), \\
& L^{\prime \prime}=2^{r\left(m_{2}+2\right)-1}(1+Q)\left[\left(D_{b}^{\prime}\right)^{r / 2} 2^{3 r / 2-1}\left(1+2^{r q_{b}^{\prime} / 2}\right)+\left(K_{b}^{\prime}\right)^{r}\right] .
\end{aligned}
$$

The application of (15) and (16) to (10) finishes the proof, where

$$
C=2^{2 r-2}\left\{\left(T-t_{0}\right)^{r / 2}\left[K^{\prime}+\left[\left(m_{1}+1\right) !\right]^{-r} K^{\prime \prime}\right]+c_{r}\left[L^{\prime}+\left[\left(m_{2}+1\right) !\right]^{-r} L^{\prime \prime}\right]\right\}
$$

is constant independent of both $n$ and $\delta_{n}$. $\diamond$

Note that in the previous proof the fact that the partition (4) is equidistant does not play any role. This, however, is not the case in the following proposition.

Proposition 2. Let the conditions of Proposition 1 and assumption $\mathcal{A}_{2}$ be satisfied. Then, for every $0<r \leq\left(M+2+\left[M \vee \frac{q}{2}\right]\right) p$,

$$
E\left\|x_{t}^{n}-x_{t_{k}}^{n}\right\|_{V}^{r} \leq \bar{B} \delta_{n}^{r / 2}, \quad t \in\left[t_{k}, t_{k+1}\right], \quad k \in\{0, \ldots, n-1\},
$$

where $\bar{B}$ is a positive generic constant independent of both $n$ and $\delta_{n}$.

Proof. The proof is going to be represented for $r \geq 2$ and, for $0<r<2$, the analogous analysis holds as in the proof of Proposition 1.

Let $t \in\left[t_{k}, t_{k+1}\right]$ for a fixed $k \in\{0, \ldots, n-1\}$. Define the sets $S_{1}$ and $S_{2}$ as $S_{1}=\left\{t \in\left[t_{0}-\tau, T\right] \mid t-\tau<t_{0}\right\}$ and $S_{2}=\left\{t \in\left[t_{0}-\tau, T\right] \mid t_{k}-\tau<t_{0} \leq t-\tau\right\}$. If we denote by $\chi_{S}$ the characteristic function of the set $S, S \subset\left[t_{0}-\tau, T\right]$, that is $\chi_{S}(y)=1$ if $y \in S$ and $\chi_{S}(y)=0$ if $y \notin S$, then

$$
E\left\|x_{t}^{n}-x_{t_{k}}^{n}\right\|_{V}^{r} \leq E_{1}+E_{2}+E_{3}
$$


where

$$
\begin{aligned}
& E_{1}=E \sup _{u \in\left[t_{k}-\tau, t_{0}+t_{k}-t\right]}\left|\eta\left(u+t-t_{k}-t_{0}\right)-\eta\left(u-t_{0}\right)\right|_{E}^{r} \cdot \chi_{S_{1}}(t), \\
& E_{2}=E \sup _{u \in\left[t_{0}+t_{k}-t, t_{0}\right]}\left|x^{n}\left(u+t-t_{k}\right)-\eta\left(u-t_{0}\right)\right|_{E}^{r} \cdot \chi_{S_{1} \cup S_{2}}(t), \\
& E_{3}=E \sup _{u \in\left[t_{0}, t_{k}\right]}\left|x^{n}\left(u+t-t_{k}\right)-x^{n}(u)\right|_{E}^{r}
\end{aligned}
$$

For completeness of the proof we will emphasize some pats of the proof of Proposition 2 from [16]. Almost sure continuity of the process $x^{n}$ and compactness of the segment $\left[t_{0}, t_{k}\right]$ implies that there exist $i \in\{0, \ldots, k-1\}$ and $v \in\left[t_{i}, t_{i+1}\right]$ such that $\sup _{u \in\left[t_{0}, t_{k}\right]}\left|x^{n}\left(u+t-t_{k}\right)-x^{n}(u)\right|^{r}=\mid x^{n}(v+t-$ $\left.t_{k}\right)+\left.x^{n}(v)\right|^{r}$. Differentiating the cases where $v+t-t_{k}$ belongs to $\left[t_{i}, t_{i+1}\right]$ or $\left[t_{i+1}, t_{i+2}\right]$, by adding and subtracting the terms $x^{n}\left(t_{i}\right)$, that is, $x^{n}\left(t_{i}\right)$ and $x^{n}\left(t_{i+1}\right)$, respectively, by applying (8) and Proposition 1, it follows that

$$
E_{3} \leq 3^{r} C \delta_{n}^{r / 2} .
$$

Since

$$
\begin{gathered}
E_{2} \leq 2^{r-1}\left[E \sup _{u \in\left[t_{0}+t_{k}-t, t_{0}\right]}\left|x^{n}\left(u+t-t_{k}\right)-x^{n}\left(t_{0}\right)\right|_{E}^{r}\right. \\
\left.+E \sup _{u \in\left[t_{0}+t_{k}-t, t_{0}\right]}\left|\eta(0)-\eta\left(u-t_{0}\right)\right|_{E}^{r}\right],
\end{gathered}
$$

by applying the assumption $\mathcal{A}_{2}$ it follows that

$$
\left|\eta(0)-\eta\left(u-t_{0}\right)\right|_{E}^{r} \leq D^{\prime r / 2}\left(1+\left|u-t_{0}\right|^{q^{\prime}}\right)^{r / 2}\left|u-t_{0}\right|^{r},
$$

where $u-t_{0}$ belongs to the segment $[-\tau, 0]$, more precisely, $u-t_{0} \in\left[t_{k}-t, 0\right]$. Because of that

$$
\left|\eta(0)-\eta\left(u-t_{0}\right)\right|_{E}^{r} \leq D^{\prime r / 2}\left(1+\tau^{q^{\prime}}\right)^{r / 2}(1 \wedge \tau)^{r / 2} \delta_{n}^{r / 2} .
$$

Combining the previous relation with Proposition 1 leads to

$$
E_{2} \leq 2^{r-1}\left[C+D^{\prime r / 2}\left(1+\tau^{q^{\prime}}\right)^{r / 2}(1 \wedge \tau)^{r / 2}\right] \delta_{n}^{r / 2} .
$$

Similarly, for estimating the expression $E_{1}$, we get

$$
\begin{aligned}
& \left|\eta\left(u+t-t_{k}-t_{0}\right)-\eta\left(u-t_{0}\right)\right|_{E}^{r} \\
& \quad \leq D^{\prime r / 2}\left(1+\left|u+t-t_{k}-t_{0}\right|^{q^{\prime}}+\left|u-t_{0}\right|^{q^{\prime}}\right)^{r / 2}\left|t-t_{k}\right|^{r} .
\end{aligned}
$$


Both $u+t-t_{k}-t_{0}$ and $u-t_{0}$ belong to the segment $[-\tau, 0]$, so

$$
E_{1} \leq D^{\prime r / 2}\left(1+2 \tau^{q^{\prime}}\right)^{r / 2}(1 \wedge \tau)^{r / 2} \delta_{n}^{r / 2} .
$$

Finally, the relations (18), (19) and (20), together with (17), imply straightforward the proof of this proposition with the positive constant

$\bar{B}=3^{r} C+2^{r-1}\left[C+D^{\prime r / 2}\left(1+\tau^{q^{\prime}}\right)^{r / 2}(1 \wedge \tau)^{r / 2}\right]+D^{\prime r / 2}\left(1+2 \tau^{q^{\prime}}\right)^{r / 2}(1 \wedge \tau)^{r / 2}$, independent of $n$ and $\delta_{n} . \diamond$

In the next theorem, the rate of convergence for the analytic method under consideration is established. It is shown that if the degrees of the Taylor approximations of the functionals $a$ and $b$ increase, the rate of the closeness between solutions $x$ and $x^{n}$ increases as well in the sense of $L^{p}$-norm.

Theorem 2.1. Let us assume that all assumptions of Proposition 2 hold, let $x$ be the solution of Eq. (3) with the initial condition (2) and $x^{n}$ be the solutions of Eqs. (5). Then, for $p>0$

$$
E \sup _{t \in\left[t_{0}-\tau, T\right]}\left|x(t)-x^{n}(t)\right|_{E}^{p} \leq H \delta_{n}^{(m+1) p / 2},
$$

where $m=m_{1} \wedge m_{2}$ and $H$ is a generic constant independent of $n$ and $\delta_{n}$.

Proof. Analogously to the proofs of Propositions 1 and 2, only the case when $p \geq 2$ is going to be considered.

Let $j$ be the biggest integer such that $t \in\left[t_{j}, T\right]$. Using elementary inequalities such as Hölder inequality with the conjugate exponents $(p, p /(p-1))$ for the appropriate Lebesgue integral, Hölder inequality with the conjugate exponents $(p / 2, p /(p-2))$, Burkholder-Davis-Gundy inequality (for $p>2$ ), Itô isometry, Doob martingale inequality (for $p=2$ ) for the appropriate Itô integral and Fubini theorem, we get

$$
\begin{aligned}
E & \sup _{s \in\left[t_{0}-\tau, t\right]}\left|x(s)-x^{n}(s)\right|_{E}^{p} \\
\leq & 2^{p-1}\left(T-t_{0}\right)^{p-1} \sum_{k=0}^{j-1} \int_{t_{k}}^{t_{k+1}} E\left|a\left(x_{u}, u\right)-A\left(x_{u}^{n}, u ; x_{t_{k}}^{n}\right)\right|_{E}^{p} d u \\
& +2^{p-1}\left(T-t_{0}\right)^{p-1} \int_{t_{j}}^{t} E\left|a\left(x_{u}, u\right)-A\left(x_{u}^{n}, u ; x_{t_{k}}^{n}\right)\right|_{E}^{p} d u \\
& +2^{p-1} c_{p}\left(T-t_{0}\right)^{p / 2-1} \sum_{k=0}^{j-1} \int_{t_{k}}^{t_{k+1}} E\left|b\left(x_{u}, u\right)-B\left(x_{u}^{n}, u ; x_{t_{k}}^{n}\right)\right|_{E}^{p} d u \\
& +2^{p-1} c_{p}\left(T-t_{0}\right)^{p / 2-1} \int_{t_{j}}^{t} E\left|b\left(x_{u}, u\right)-B\left(x_{u}^{n}, u ; x_{t_{k}}^{n}\right)\right|_{E}^{p} d u
\end{aligned}
$$


It is easy to see that, for every $t \in\left[t_{k}, t_{k+1}\right], k \in\{0,1, \ldots, j\}$,

$$
\begin{aligned}
& \int_{t_{k}}^{t} E\left|a\left(x_{u}, u\right)-A\left(x_{u}^{n}, u ; x_{t_{k}}^{n}\right)\right|_{E}^{p} d u \\
& =\int_{t_{k}}^{t} E\left|a\left(x_{u}, u\right)-a\left(x_{u}^{n}, u\right)+a\left(x_{u}^{n}, u\right)-A\left(x_{u}^{n}, u ; x_{t_{k}}^{n}\right)\right|_{E}^{p} d u \\
& \leq 2^{p-1}\left(\int_{t_{k}}^{t} E\left|a\left(x_{u}, u\right)-a\left(x_{u}^{n}, u\right)\right|_{E}^{p} d u+\int_{t_{k}}^{t} E\left|a\left(x_{u}^{n}, u\right)-A\left(x_{u}^{n}, u ; x_{t_{k}}^{n}\right)\right|_{E}^{p} d u\right) .
\end{aligned}
$$

For the first summand in (22), the polynomial condition $\mathcal{A}_{5}$ and CauchySchwarz-Bunyakovsky inequality imply

$$
\begin{aligned}
& \int_{t_{k}}^{t} E\left|a\left(x_{u}, u\right)-a\left(x_{u}^{n}, u\right)\right|_{E}^{p} d u \\
& \leq \int_{t_{k}}^{t} E\left[D_{a}\left(1+\left\|x_{u}\right\|_{V}^{q_{a}}+\left\|x_{u}^{n}\right\|_{V}^{q_{a}}\right)\left\|x_{u}-x_{u}^{n}\right\|_{V}^{2}\right]^{p / 2} d u \\
& \leq D_{a}^{p / 2} \int_{t_{k}}^{t}\left\{E\left[\left(1+\left\|x_{u}\right\|_{V}^{q_{a}}+\left\|x_{u}^{n}\right\|_{V}^{q_{a}}\right)\left\|x_{u}-x_{u}^{n}\right\|_{V}\right]^{p}\right\}^{1 / 2}\left\{E\left\|x_{u}-x_{u}^{n}\right\|_{V}^{p}\right\}^{1 / 2} d u
\end{aligned}
$$

By using the inequality (8), Cauchy-Schwarz-Bunyakovsky inequality and $\mathcal{A}_{6}$, we compute

$$
\begin{aligned}
& E\left[\left(1+\left\|x_{u}\right\|_{V}^{q_{a}}+\left\|x_{u}^{n}\right\|_{V}^{q_{a}}\right)\left\|x_{u}-x_{u}^{n}\right\|_{V}\right]^{p} \\
& \leq 3^{p-1} 2^{p-1} E\left[\left(1+\left\|x_{u}\right\|_{V}^{p q_{a}}+\left\|x_{u}^{n}\right\|_{V}^{p q_{a}}\right)\left(\left\|x_{u}\right\|_{V}^{p}+\left\|x_{u}^{n}\right\|_{V}^{p}\right)\right] \\
& \leq 6^{p-1}\left[E\left\|x_{u}\right\|_{V}^{p}+E\left\|x_{u}^{n}\right\|_{V}^{p}+E\left\|x_{u}\right\|_{V}^{p\left(1+q_{a}\right)}+E\left\|x_{u}^{n}\right\|_{V}^{p\left(1+q_{a}\right)}\right. \\
& \left.\quad \quad+\left(E\left\|x_{u}\right\|_{V}^{2 p}\right)^{1 / 2}\left(E\left\|x_{u}^{n}\right\|_{V}^{2 p q_{a}}\right)^{1 / 2}+\left(E\left\|x_{u}\right\|_{V}^{2 p q_{a}}\right)^{1 / 2}\left(E\left\|x_{u}^{n}\right\|_{V}^{2 p}\right)^{1 / 2}\right] \\
& \quad \leq 6^{p}(1+Q) .
\end{aligned}
$$

Then, (23) becomes

$$
\int_{t_{k}}^{t} E\left|a\left(x_{u}, u\right)-a\left(x_{u}^{n}, u\right)\right|_{E}^{p} d u \leq D_{a}^{p / 2}\left(6^{p}(1+Q)\right)^{1 / 2} \int_{t_{k}}^{t}\left(E\left\|x_{u}-x_{u}^{n}\right\|_{V}^{p}\right)^{1 / 2} d u .
$$

The second summand in (22) is estimated applying the condition $\mathcal{A}_{1}$ and 
relation (7), such that

$$
\begin{aligned}
& \int_{t_{k}}^{t} E\left|a\left(x_{u}^{n}, u\right)-A\left(x_{u}^{n}, u ; x_{t_{k}}^{n}\right)\right|_{E}^{p} d u \\
& \equiv \int_{t_{k}}^{t} E\left|\frac{a_{\left(x_{t_{k}}^{n}+\bar{\theta} \Delta_{u}^{n}, u\right)}^{\left(m_{1}+1\right)}\left(\Delta_{u}^{n}, \ldots, \Delta_{u}^{n}\right)}{\left(m_{1}+1\right) !}\right|_{E}^{p} d u \\
& \leq\left[\left(m_{1}+1\right) !\right]^{-p} \int_{t_{k}}^{t} E\left(\sup _{\bar{\theta} \in[0,1]}\left\|a_{\left(x_{t_{k}}^{n}+\bar{\theta} \Delta_{u}^{n}, u\right)}^{\left(m_{1}+1\right)}\right\|_{M, m_{1}+1}^{p}\left\|\Delta_{u}^{n}\right\|_{V}^{\left(m_{1}+1\right) p}\right) d u,
\end{aligned}
$$

where $\Delta_{u}^{n}=x_{s}^{n}-x_{t_{k}}^{n}$, as in Proposition 2. The application of the relation (8) multiple times, $\mathcal{A}_{4}$ and $\mathcal{A}_{5}$, yields

$$
\begin{aligned}
& \sup _{\bar{\theta} \in[0,1]}\left\|a_{\left(x_{t_{k}}^{n}+\bar{\theta} \Delta_{u}^{n}, u\right)}^{\left(m_{1}+1\right)}\right\|_{M, m_{1}+1}^{p} \\
& \leq 2^{p-1}\left\{\left\|a_{(0, u)}^{\left(m_{1}+1\right)}\right\|_{M, m_{1}+1}^{p}+\sup _{\theta \in[0,1]}\left\|a_{\left(x_{t_{k}}^{n}+\bar{\theta} \Delta_{u}^{n}, u\right)}^{\left(m_{1}+1\right)}-a_{(0, u)}^{\left(m_{1}+1\right)}\right\|_{M, m_{1}+1}^{p}\right\} \\
& \leq 2^{p-1}\left\{\left(K_{a}^{\prime}\right)^{p}+\sup _{\theta \in[0,1]}\left[D_{a}^{\prime}\left(1+\left\|x_{t_{k}}^{n}+\bar{\theta} \Delta_{u}^{n}\right\|_{V}^{q_{a}^{\prime}}\right)\left\|x_{t_{k}}^{n}+\bar{\theta} \Delta_{u}^{n}\right\|_{V}^{2}\right]^{p / 2}\right\} \\
& \leq 2^{p-1}\left\{\left(K_{a}^{\prime}\right)^{p}+\left(D_{a}^{\prime}\right)^{p / 2} 2^{3 p / 2-2}\left[\sup _{\bar{\theta} \in[0,1]}\left(\left\|x_{t_{k}}^{n}\right\|_{V}^{p}+\bar{\theta}^{p}\left\|\Delta_{u}^{n}\right\|_{V}^{p}\right)\right.\right. \\
& \left.\left.+2^{p q_{a}^{\prime} / 2} \sup _{\bar{\theta} \in[0,1]}\left(\left\|x_{t_{k}}^{n}\right\|_{V}^{p\left(1+q_{a}^{\prime} / 2\right)}+\bar{\theta}^{p\left(1+q_{a}^{\prime} / 2\right)}\left\|\Delta_{u}^{n}\right\|_{V}^{p\left(1+q_{a}^{\prime} / 2\right)}\right)\right]\right\} \\
& \leq 2^{p-1}\left\{\left(K_{a}^{\prime}\right)^{p}+\left(D_{a}^{\prime}\right)^{p / 2} 2^{3 p / 2-2}\left[\left\|x_{t_{k}}^{n}\right\|_{V}^{p}+\left\|\Delta_{u}^{n}\right\|_{V}^{p}\right.\right. \\
& \left.\left.+2^{p q_{a}^{\prime} / 2}\left(\left\|x_{t_{k}}^{n}\right\|_{V}^{p\left(1+q_{a}^{\prime} / 2\right)}+\left\|\Delta_{u}^{n}\right\|_{V}^{p\left(1+q_{a}^{\prime} / 2\right)}\right)\right]\right\} .
\end{aligned}
$$

Then, (25) becomes

$$
\begin{aligned}
& \int_{t_{k}}^{t} E\left|a\left(x_{u}^{n}, u\right)-A\left(x_{u}^{n}, u ; x_{t_{k}}^{n}\right)\right|_{E}^{p} d u \\
& \leq \frac{2^{p-1}}{\left[\left(m_{1}+1\right) !\right]^{p}} \\
& \quad \cdot \int_{t_{k}}^{t} E\left\{\left[\left(K_{a}^{\prime}\right)^{p}+\left(D_{a}^{\prime}\right)^{p / 2} 2^{3 p / 2-2}\left(\left\|x_{t_{k}}^{n}\right\|^{p}+\left\|\Delta_{u}^{n}\right\|^{p}\right.\right.\right. \\
& \left.\left.\left.\quad+2^{p q_{a}^{\prime} / 2}\left(\left\|x_{t_{k}}^{n}\right\|^{p\left(1+q_{a}^{\prime} / 2\right)}+\left\|\Delta_{u}^{n}\right\|^{p\left(1+q_{a}^{\prime} / 2\right)}\right)\right)\right]\left\|\Delta_{u}^{n}\right\|^{\left(m_{1}+1\right) p}\right\} d u
\end{aligned}
$$




$$
\begin{aligned}
\equiv \int_{t_{k}}^{t} E[ & K_{1}\left\|\Delta_{u}^{n}\right\|^{\left(m_{1}+1\right) p}+K_{2}\left\|\Delta_{u}^{n}\right\|^{\left(m_{1}+2\right) p}+K_{3}\left\|\Delta_{u}^{n}\right\|^{\left(m_{1}+2+q_{a}^{\prime} / 2\right) p} \\
& \left.\quad+K_{4}\left\|x_{t_{k}}^{n}\right\|^{p}\left\|\Delta_{u}^{n}\right\|^{\left(m_{1}+1\right) p}+K_{5}\left\|x_{t_{k}}^{n}\right\|^{p\left(1+q_{a}^{\prime} / 2\right)}\left\|\Delta_{u}^{n}\right\|^{\left(m_{1}+1\right) p}\right] d u
\end{aligned}
$$

where

$$
\begin{aligned}
& K_{1}=\frac{2^{p-1}\left(K_{a}^{\prime}\right)^{p}}{\left[\left(m_{1}+1\right) !\right]^{p}}, \quad K_{2}=K_{4}=\frac{2^{5 p / 2-3}\left(D_{a}^{\prime}\right)^{p / 2}}{\left[\left(m_{1}+1\right) !\right]^{p}} \\
& K_{3}=K_{5}=\frac{2^{p\left(5 / 2+q_{a}^{\prime} / 2\right)-3}\left(D_{a}^{\prime}\right)^{p / 2}}{\left[\left(m_{1}+1\right) !\right]^{p}}
\end{aligned}
$$

Finally, via (8), the Cauchy-Schwarz-Bunyakovsky inequality, assumption $\mathcal{A}_{6}$ and Proposition 2, (26) implies

$$
\begin{aligned}
& \int_{t_{k}}^{t} E\left|a\left(x_{u}^{n}, u\right)-A\left(x_{u}^{n}, u ; x_{t_{k}}^{n}\right)\right|_{E}^{p} d u \\
& \leq \int_{t_{k}}^{t}\left\{E \left[K_{1}\left\|\Delta_{u}^{n}\right\|^{\left(m_{1}+1\right) p}+K_{2}\left\|\Delta_{u}^{n}\right\|^{\left(m_{1}+2\right) p}+K_{3}\left\|\Delta_{u}^{n}\right\|^{\left.\left(m_{1}+2+q_{a}^{\prime} / 2\right) p\right]}\right.\right. \\
& \quad+K_{4}\left(E\left\|x_{t_{k}}^{n}\right\|^{2 p}\right)^{1 / 2}\left(E\left\|\Delta_{u}^{n}\right\|^{2\left(m_{1}+1\right) p}\right)^{1 / 2} \\
& \quad+K_{5}\left(E\left\|x_{t_{k}}^{n}\right\|^{2 p\left(1+q_{a}^{\prime} / 2\right)}\right)^{1 / 2}\left(E\left\|\Delta_{u}^{n}\right\|^{\left.\left.2\left(m_{1}+1\right) p\right)^{1 / 2}\right\}}\right\} d u \\
& \leq \int_{t_{k}}^{t}\left\{E\left[K_{1}\left\|\Delta_{u}^{n}\right\|^{\left(m_{1}+1\right) p}+K_{2}\left\|\Delta_{u}^{n}\right\|^{\left(m_{1}+2\right) p}+K_{3}\left\|\Delta_{u}^{n}\right\|^{\left(m_{1}+2+q_{a}^{\prime} / 2\right) p}\right]\right. \\
& \left.\quad+(1+Q)^{1 / 2}\left[K_{4}\left(E\left\|\Delta_{u}^{n}\right\|^{2\left(m_{1}+1\right) p}\right)^{1 / 2}+K_{5}\left(E\left\|\Delta_{u}^{n}\right\|^{2\left(m_{1}+1\right) p}\right)^{1 / 2}\right]\right\} d u \\
& \leq\left[\bar{B}\left(K_{1} \delta_{n}^{\left(m_{1}+1\right) p / 2}+K_{2} \delta_{n}^{\left(m_{1}+2\right) p / 2}+K_{3} \delta_{n}^{\left(m_{1}+2+q_{a}^{\prime} / 2\right) p / 2}\right)\right. \\
& \left.+\left(K_{4}+K_{5}\right)((1+Q) \bar{B})^{1 / 2} \delta_{n}^{\left(m_{1}+1\right) p / 2}\right]\left(t-t_{k}\right) .
\end{aligned}
$$

Since $\left(m_{1}+1\right) p / 2=\left[\left(m_{1}+1\right) p / 2\right] \wedge\left[\left(m_{1}+2\right) p / 2\right] \wedge\left[\left(m_{1}+2+q_{a}^{\prime} / 2\right) p / 2\right]$ and $\delta_{n}$ is less than 1 , then

$$
\begin{aligned}
& \int_{t_{k}}^{t} E\left|a\left(x_{u}^{n}, u\right)-A\left(x_{u}^{n}, u ; x_{t_{k}}^{n}\right)\right|_{E}^{p} d u \\
& \quad \leq\left[\bar{B}\left(K_{1}+K_{2}+K_{3}\right)+((1+Q) \bar{B})^{1 / 2}\left(K_{4}+K_{5}\right)\right] \delta_{n}^{\left(m_{1}+1\right) p / 2}\left(t-t_{k}\right) .
\end{aligned}
$$


In view of (24) and (28), from the inequality (22) we get

$$
\begin{aligned}
& \int_{t_{k}}^{t} E \mid a\left(x_{u}, u\right)-\left.A\left(x_{u}^{n}, u ; x_{t_{k}}^{n}\right)\right|_{E} ^{p} d u \\
& \leq 2^{p-1}\left[D_{a}^{p / 2}\left(6^{p}(1+Q)\right)^{1 / 2} \int_{t_{k}}^{t}\left(E\left\|x_{u}-x_{u}^{n}\right\|_{V}^{p}\right)^{1 / 2} d u\right. \\
&\left.\quad+\left[\bar{B}\left(K_{1}+K_{2}+K_{3}\right)+((1+Q) \bar{B})^{1 / 2}\left(K_{4}+K_{5}\right)\right] \delta_{n}^{\left(m_{1}+1\right) p / 2}\left(t-t_{k}\right)\right] .
\end{aligned}
$$

Analogously, we can show similar results for functionals $b$ and $B$ for every $k \in\{0,1, \ldots, j\}$ and $t \in\left[t_{k}, t_{k+1}\right]$, that is,

$$
\begin{aligned}
\int_{t_{k}}^{t} E \mid & b\left(x_{u}, u\right)-\left.B\left(x_{u}^{n}, u ; x_{t_{k}}^{n}\right)\right|_{E} ^{p} d u \\
\leq 2^{p-1}[ & D_{b}^{p / 2}\left(6^{p}(1+Q)\right)^{1 / 2} \int_{t_{k}}^{t}\left(E\left\|x_{u}-x_{u}^{n}\right\|_{V}^{p}\right)^{1 / 2} d u \\
& \left.+\left[\bar{B}\left(K_{1}^{\prime}+K_{2}^{\prime}+K_{3}^{\prime}\right)+((1+Q) \bar{B})^{1 / 2}\left(K_{4}^{\prime}+K_{5}^{\prime}\right)\right] \delta_{n}^{\left(m_{2}+1\right) p / 2}\left(t-t_{k}\right)\right]
\end{aligned}
$$

where the constants $K_{i}^{\prime}$ have the same form as $K_{i}, i=1,2, \ldots, 5$ in (27), but $m_{1}, K_{a}^{\prime}, D_{a}^{\prime}$ and $q_{a}^{\prime}$ are replaced by $m_{2}, K_{b}^{\prime}, D_{b}^{\prime}$ and $q_{b}^{\prime}$, respectively.

Inequalities (29) and (30) applied to (21) give

$$
\begin{aligned}
& E \sup _{s \in\left[t_{0}-\tau, t\right]}\left|x(s)-x^{n}(s)\right|_{E}^{p} \\
& \leq 2^{5 p / 2-2} 3^{p / 2}(1+Q)^{1 / 2}\left(T-t_{0}\right)^{p / 2-1}\left[\left(T-t_{0}\right)^{p / 2} D_{a}^{p / 2}+c_{p} D_{b}^{p / 2}\right] \\
& \cdot \int_{t_{0}}^{t}\left(E\left\|x_{u}-x_{u}^{n}\right\|_{V}^{p}\right)^{1 / 2} d u \\
&+ 2^{2 p-2}\left(T-t_{0}\right)^{p / 2-1}\left[\left(T-t_{0}\right)^{p / 2}+c_{p}\right] \bar{B}^{1 / 2}\left(t-t_{0}\right) \\
& \cdot\left\{\left[\bar{B}^{1 / 2}\left(K_{1}+K_{2}+K_{3}\right)+(1+Q)^{1 / 2}\left(K_{4}+K_{5}\right)\right] \delta_{n}^{\left(m_{1}+1\right) p / 2}\right. \\
&\left.\quad+\left[\bar{B}^{1 / 2}\left(K_{1}^{\prime}+K_{2}^{\prime}+K_{3}^{\prime}\right)+(1+Q)^{1 / 2}\left(K_{4}^{\prime}+K_{5}^{\prime}\right)\right] \delta_{n}^{\left(m_{2}+1\right) p / 2}\right\} \\
& \leq L_{1} \int_{t_{0}}^{t}\left(E\left\|x_{u}-x_{u}^{n}\right\|_{V}^{p}\right)^{1 / 2} d u+L_{2} \delta_{n}^{(m+1) p / 2} \\
& \leq L_{1} \int_{t_{0}}^{t}\left(E \sup _{s \in\left[t_{0}-\tau, u\right]}\left|x(s)-x^{n}(s)\right|_{E}^{p}\right)^{1 / 2} d u+L_{2} \delta_{n}^{(m+1) p / 2},
\end{aligned}
$$


since $\delta_{n}$ is less than 1 , where

$$
\begin{gathered}
L_{1}=2^{5 / 2 p-2} 3^{p / 2}(1+Q)^{1 / 2}\left(T-t_{0}\right)^{p / 2-1}\left[\left(T-t_{0}\right)^{p / 2} D_{a}^{p / 2}+c_{p} D_{b}^{p / 2}\right] \\
\begin{array}{c}
L_{2}=2^{2 p-2}\left(T-t_{0}\right)^{p / 2}\left[\left(T-t_{0}\right)^{p / 2}+c_{p}\right] \bar{B}^{1 / 2} \\
\cdot\left[\bar{B}^{1 / 2}\left(K_{1}+K_{2}+K_{3}\right)+(1+Q)^{1 / 2}\left(K_{4}+K_{5}\right)\right. \\
\left.\quad+\bar{B}^{1 / 2}\left(K_{1}^{\prime}+K_{2}^{\prime}+K_{3}^{\prime}\right)+(1+Q)^{1 / 2}\left(K_{4}^{\prime}+K_{5}^{\prime}\right)\right] .
\end{array}
\end{gathered}
$$

The relation (31) has the form (9) and the Bihari-type inequality (Theorem 1.1) applied to (31) finishes the proof if all of its assumptions hold. Indeed, let the function $\varphi: \mathbb{R}^{+} \rightarrow \mathbb{R}^{+}$be defined as $\varphi(y)=y^{1 / 2}$ and it is nondecreasing, positive for positive arguments and satisfies the relation $\varphi(y) \leq \alpha \varphi(y / \alpha)$, for every $y \in[0, \infty)$ and $\alpha \geq 1$. Also, $E \sup _{s \in\left[t_{0}-\tau, t\right]}\left|x(s)-x^{n}(s)\right|_{E}^{p}$, considered as the function of the argument $t, t \in\left[t_{0}, T\right]$, is a nonnegative and continuous function. Then, from the application of Theorem 1.1 it follows that

$$
E \sup _{s \in\left[t_{0}-\tau, t\right]}\left|x(s)-x^{n}(s)\right|_{E}^{p} \leq L_{2} \delta_{n}^{(m+1) p / 2} \frac{1}{4}\left(2+L_{1} T\right)^{2}=H \delta_{n}^{(m+1) p / 2},
$$

where $G(y)=\int_{0}^{y} \frac{d s}{\varphi(s)} d s=2 y^{1 / 2}$, for $y>0$, and its inverse function is $G^{-1}(y)=$ $\frac{1}{4} y^{2}$. The constant $H=\frac{L_{2}}{4}\left(2+L_{1} T\right)^{2}$ is independent of $\delta_{n}$ and $n$. $\diamond$

The following theorem is proved analogously to Theorem 2 from [16]. The proof is based on the application of the Borel-Cantelli lemma. Almost sure convergence of the sequence of the solutions to approximate Eqs. (5) to the solution of the initial Eq. (3) is established.

Theorem 2.2. Let the assumptions of Theorem 1 be satisfied. Then, the sequence $\left(x^{n}\right)_{n \in \mathbb{N}}$ of the solutions of Eqs. (5) converges almost surely to the solution $x$ of Eq. (3).

\section{Example}

The next example illustrates the previous theoretical findings.

Let us consider an one-dimensional $(d=1)$ SFDE

$$
\begin{aligned}
d x(t)=[ & \left.-\alpha(t) C(x(t))+0.5 \beta(t) \sin \left(2 \int_{-\tau}^{0} K(\theta) x_{t}(\theta) d \theta\right)\right] d t \\
& +a_{1}(t) \sin \left(\int_{-\tau}^{0} K(\theta) x_{t}(\theta) d \theta\right) d w(t), \quad t \in\left[t_{0}, T\right]
\end{aligned}
$$

with the initial condition $x_{0}=\eta=\{\theta \mid \theta \in[-\tau, 0]\}$ independent of the onedimensional $\left(d_{1}=1\right)$ Brownian motion $w$, where $C(x)=\frac{1}{2} \int_{0}^{x^{2}} \frac{\cos y}{\sqrt{y}} d y, x \in R$ 
is Fresnel integral and $\alpha, \beta, a_{1}$ are nonnegative, continuous functions from $[0, T]$ to $\mathbb{R}$, and, therefore, bounded. Let us introduce those boundaries: for every $t \in\left[t_{0}, T\right], \alpha(t) \leq \bar{\alpha}, \beta(t) \leq \bar{\beta}$ and $a_{1}(t) \leq \bar{a}_{1}$.

The drift coefficient

$$
a\left(x_{t}, t\right)=-\alpha(t) C(x(t))+0.5 \beta(t) \sin \left(2 \int_{-\tau}^{0} K(\theta) x_{t}(\theta) d \theta\right)
$$

and diffusion coefficient

$$
b\left(x_{t}, t\right)=a_{1}(t) \sin \left(\int_{-\tau}^{0} K(\theta) x_{t}(\theta) d \theta\right)
$$

are considered for an arbitrary but fixed argument $t \in\left[t_{0}, T\right]$. In other words, these drift and diffusion coefficients could be represented as the functionals $a_{t} \equiv a=f \circ \underline{h}+A_{1}: V \rightarrow \mathbb{R}$ and $b_{t} \equiv b=g \circ \underline{h}: V \rightarrow \mathbb{R}$, where the functional $\underline{h}: V \rightarrow \mathbb{R}$ is defined as

$$
\underline{h}(v)=\int_{-\tau}^{0} K(\theta) v(\theta) d \theta, \quad v \in V,
$$

where the continuous function $K:[-\tau, 0] \rightarrow \mathbb{R}^{+}$satisfies $\int_{-\tau}^{0} K(\theta) d \theta=1$, functions $f, g: \mathbb{R} \rightarrow \mathbb{R}$ are defined as $f(x)=0.5 \beta(t) \sin (2 x)$ and $g(x)=a_{1}(t) \sin x$, $x \in \mathbb{R}$ and functional $A_{1}: V \rightarrow \mathbb{R}$ is defined as $A_{1}(v)=\alpha(t) C(v(0)), v \in V$. For the purposes of this example, we choose

$$
K(\theta)=\frac{1}{\tau}, \quad \theta \in[-\tau, 0] .
$$

Both the drift and diffusion coefficients from (32) are continuously-differentiable (in the first argument) and globally Lipschitz continuous. More precisely, there exist positive constants $L_{a}=2\left(\bar{\alpha}^{2}+\bar{\beta}^{2}\right)$ and $L_{b}=\bar{a}_{1}^{2}$ such that, for every $u, v \in V$ and $t \in\left[t_{0}, T\right]$,

$$
|a(u, t)-a(v, t)|^{2} \leq L_{a}\|u-v\|_{V}^{2}, \quad|b(u, t)-b(v, t)|^{2} \leq L_{b}\|u-v\|_{V}^{2} .
$$

Let us consider the first Fréchet derivatives of the coefficients $a \equiv a_{t}$ and $b \equiv b_{t}$. It can be easily seen that the real functions of real argument $f$ and $g$ are differentiable arbitrary many times in any real point $x$, so, there exist Fréchet derivatives $f_{x}^{\prime}$ and $g_{x}^{\prime}$ in $\mathcal{L}(\mathbb{R}, \mathbb{R})$ of the functions $f$ and $g$, respectively, in the point $x$. It is easy to verify (see $[4,5]$, for example) that for any $y \in \mathbb{R}$, $f_{x}^{\prime}(y)=f^{\prime}(x) \cdot y \in \mathbb{R}$ and $g_{x}^{\prime}(y)=g^{\prime}(x) \cdot y \in \mathbb{R}$, where the derivatives on the left side of these equalities represent the Fréchet derivatives and the ones 
on the right side represent the ordinary derivatives of real functions with real arguments.

It is obvious that $\underline{h}$ belongs to the space $\mathcal{L}(V, \mathbb{R})$, that is, it is bounded linear operator, such that, for every $u \in V$, there exists the first Fréchet derivative $\underline{h}_{u}^{\prime}=\underline{h} \in \mathcal{L}(V, \mathbb{R})$ in the point $u$. Also, there exists the second Fréchet derivative in $u, \underline{h}_{u}^{\prime \prime}=\mathbf{0} \in \mathcal{L}(V, \mathcal{L}(V, \mathbb{R})) \cong \mathcal{T}\left(V^{2}, \mathbb{R}\right)$, which is not difficult to prove (see $[4,5])$.

Let $u \in V$ be arbitrary. To find $A_{1 u}^{\prime}$ explicitly, if it exists, we make the difference

$$
A_{1}(u+h)-A_{1}(u)=L_{A_{1}} h+R_{A_{1}}(u, h), \quad h \in V .
$$

If we can make this decomposition such that $\left|R_{A_{1}}(u, h)\right| /\|h\|_{V} \rightarrow 0$ as $\|h\|_{V} \rightarrow$ 0 and $L_{A_{1}} \in \mathcal{L}(V, \mathbb{R})$, then $L_{A_{1}}=A_{1 u}^{\prime}$. It is easy to verify that there exists first Fréchet derivative $A_{1 u}^{\prime} \in \mathcal{L}(V, \mathbb{R})$ of $A_{1}$ in arbitrary $u \in V$ and it is defined as $A_{1 u}^{\prime}(v)=-\alpha(t) \cos \left(u^{2}(0)\right) v(0)$ for every $v \in V$.

In a view of the chain rule ([5], p. 171, for example) there exists first Fréchet

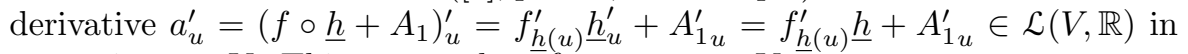
any point $u \in V$. This means that, for every $v \in V$

$$
\begin{aligned}
a_{u}^{\prime}(v) & =f^{\prime}(\underline{h}(u)) \underline{h}(v)+A_{1 u}^{\prime}(v) \\
& =-\alpha(t) \cos \left(u^{2}(0)\right) v(0)+\beta(t) \cos \left(2 \int_{-\tau}^{0} K(\theta) u(\theta) d \theta\right) \int_{-\tau}^{0} K(\theta) v(\theta) d \theta .
\end{aligned}
$$

Also, $b_{u}^{\prime}=g_{\underline{h}(u)}^{\prime} \underline{h} \in \mathcal{L}(V, \mathbb{R})$ in every $u \in V$. In other words,

$$
b_{u}^{\prime}(v)=g^{\prime}(\underline{h}(u)) \underline{h}(v)=a_{1}(t) \cos \left(\int_{-\tau}^{0} K(\theta) u(\theta) d \theta\right) \int_{-\tau}^{0} K(\theta) v(\theta) d \theta .
$$

If there exists the second Fréchet derivative of $a: V \rightarrow \mathbb{R}$ in $u \in V$, then $a_{u}^{\prime \prime} \in \mathcal{L}(V, \mathcal{L}(V, \mathbb{R}))$. The second Fréchet derivative of $a$ in $u$ is actually the first Fréchet derivative of $v \mapsto a_{v}^{\prime}$ in $u \in V$, that is, the first Fréchet derivative of the function $c: V \rightarrow \mathcal{L}(V, \mathbb{R})$ defined as $c(v)=a_{v}^{\prime}$, since the first Fréchet derivative of $a$ exists in every point of the vector space $V$. To find $a_{u}^{\prime \prime}$ explicitly, we make the difference

$$
c(u+h)-c(u)=L_{c} h+R_{c}(u, h), \quad h \in V .
$$

If we can make this decomposition such that $\left\|R_{c}(u, h)\right\|_{\mathcal{L}(V, \mathbb{R})} /\|h\|_{V} \rightarrow 0$ as $\|h\|_{V} \rightarrow 0$ and $L_{c} \in \mathcal{L}(V, \mathcal{L}(V, \mathbb{R}))$, then $L_{c}=c_{u}^{\prime}=a_{u}^{\prime \prime} \in \mathcal{L}(V, \mathcal{L}(V, \mathbb{R}))$.

It turns out that for every $v_{1}, v_{2} \in V$

$$
a_{u}^{\prime \prime}\left(v_{1}\right)\left(v_{2}\right)=2 \alpha(t) u(0) \sin u^{2}(0) v_{1}(0) v_{2}(0)-2 \beta(t) \sin (2 \underline{h}(u)) \underline{h}\left(v_{1}\right) \underline{h}\left(v_{2}\right) \in \mathbb{R} .
$$


Similarly can be done for $b_{u}^{\prime \prime}$ and we get for every $v_{1}, v_{2} \in V$,

$$
b_{u}^{\prime \prime}\left(v_{1}\right)\left(v_{2}\right)=-a_{1}(t) \sin (\underline{h}(u)) \underline{h}\left(v_{1}\right) \underline{h}\left(v_{2}\right) \in \mathbb{R} .
$$

The polynomial condition $\mathcal{A}_{5}$ holds for functionals $a, b, a^{\prime \prime}$ and $b^{\prime \prime}$ with the constants

$$
\begin{aligned}
& q_{a}^{\prime}=4, D_{a}^{\prime}=16\left[\left(\bar{\alpha}^{2}+2 \bar{\beta}^{2}\right) \vee 3 \bar{\alpha}^{2}\right] ; \quad q_{b}^{\prime}=0, D_{b}^{\prime}=\bar{a}_{1}^{2} \\
& q_{a}=0, D_{a}=2\left(\bar{\alpha}^{2}+\bar{\beta}^{2}\right) ; \quad q_{b}=0, \quad D_{b}=\bar{a}_{1}^{2} .
\end{aligned}
$$

It is easy to see that conditions $\mathcal{A}_{3}$ and $\mathcal{A}_{4}$ hold for arbitrary $K_{a}, K_{b}, K_{a}^{\prime}, K_{b}^{\prime}>$ 0 since $a(0, t)=b(0, t)=a^{\prime \prime}(0, t)=b^{\prime \prime}(0, t)=0$ for every $t \in\left[t_{0}, T\right]$. The polynomial condition $\mathcal{A}_{2}$ for initial data $\eta$ is satisfied with the constants $D^{\prime}=1$ and $q^{\prime}=0$. Condition $\mathcal{A}_{6}$ holds for the solution $x$ of Eq. (32) on the basis of [14] (see Theorem 2.2, pp. 150 and Theorem 4.1, pp. 160).

It should be pointed out that the second derivative of the drift coefficient of this equation is not uniformly bounded, such that the $L^{p}$ and a.s. convergence results from [16] can not be applied. Moreover, in the sequel, we will prove the existence, uniqueness and $p$-th moment boundedness (for any $p \geq 2$ ) of the corresponding approximate solution of this equation.

Using the Taylor expansion of the first order $\left(m_{1}=m_{2}=1\right)$ near the points $x^{n}\left(t_{k}\right)$ for $k \in\{0, \ldots, n-1\}$, Eqs. (5) become

$$
x^{n}(t)=x^{n}\left(t_{k}\right)+\int_{t_{k}}^{t} A\left(x_{s}^{n}, s ; x_{t_{k}}^{n}\right) d s+\int_{t_{k}}^{t} B\left(x_{s}^{n}, s ; x_{t_{k}}^{n}\right) d w(s),
$$

$t \in\left[t_{k}, t_{k+1}\right], k \in\{0,1, \ldots, n-1\}$, where the drift and diffusion coefficients are

$$
\begin{aligned}
A\left(x_{t}^{n}, t ; x_{t_{k}}^{n}\right)= & -\alpha(t) C\left(x^{n}\left(t_{k}\right)\right)+0.5 \beta(t) \sin \left(2 \underline{h}\left(x_{t_{k}}^{n}\right)\right) \\
& -\alpha(t) \cos \left(x^{n}\left(t_{k}\right)\right)^{2}\left[x^{n}(t)-x^{n}\left(t_{k}\right)\right] \\
& +\beta(t) \cos \left(2 \underline{h}\left(x_{t_{k}}^{n}\right)\right)\left[\underline{h}\left(x_{t}^{n}\right)-\underline{h}\left(x_{t_{k}}^{n}\right)\right], \\
B\left(x_{t}^{n}, t ; x_{t_{k}}^{n}\right)= & a_{1}(t) \sin \underline{h}\left(x_{t_{k}}^{n}\right)+a_{1}(t) \cos \underline{h}\left(x_{t_{k}}^{n}\right)\left[\underline{h}\left(x_{t}^{n}\right)-\underline{h}\left(x_{t_{k}}^{n}\right)\right] .
\end{aligned}
$$

For convenience, let us introduce processes

$$
\begin{aligned}
& z^{n}(t+\theta)=\sum_{k=0}^{n-1} I_{\left[t_{k}, t_{k+1}\right)}(t) x^{n}\left(t_{k}+\theta\right), \quad \theta \in[-\tau, 0] \\
& z^{n}(t)=\sum_{k=0}^{n-1} I_{\left[t_{k}, t_{k+1}\right)}(t) x^{n}\left(t_{k}\right)
\end{aligned}
$$


and observe from (39) that $z_{t}^{n}=x_{t_{k}}^{n}, t \in\left[t_{k}, t_{k+1}\right)$. Thus, Eqs. (37) can be represented as

$$
x^{n}(t)=\eta(0)+\int_{t_{0}}^{t} A\left(s, x_{s}^{n}, z_{s}^{n}\right) d s+\int_{t_{0}}^{t} B\left(s, x_{s}^{n}, z_{s}^{n}\right) d w(s), \quad t \in\left[t_{0}, T\right],
$$

where, for any $s \in\left[t_{0}, T\right]$,

$$
\begin{aligned}
A\left(s, x_{s}^{n}, z_{s}^{n}\right)= & -\alpha(s) C\left(z^{n}(s)\right)-\alpha(s) \cos \left(z^{n}(s)\right)^{2}\left(x^{n}(s)-z^{n}(s)\right) \\
& +\frac{1}{2} \beta(s) \sin \left(2 \underline{h}\left(z_{s}^{n}\right)\right)+\beta(s) \cos \left(2 \underline{h}\left(z_{s}^{n}\right)\right)\left(\underline{h}\left(x_{s}^{n}\right)-\underline{h}\left(z_{s}^{n}\right)\right), \\
B\left(s, x_{s}^{n}, z_{s}^{n}\right)= & a_{1}(s) \sin \left(\underline{h}\left(z_{s}^{n}\right)\right)+a_{1}(s) \cos \left(\underline{h}\left(z_{s}^{n}\right)\right)\left(\underline{h}\left(x_{s}^{n}\right)-\underline{h}\left(z_{s}^{n}\right)\right) .
\end{aligned}
$$

In the sequel, we will use the Khasminskii approach to prove the existence, uniqueness and moment boundedness of the solution to Eq. (41). This approach is successfully applied in many papers, such as, for example, [20] in the context of SFDEs. Bearing in mind that coefficients of Eq. (41) satisfy the local Lipschitz condition, it follows that for the initial condition $\eta$ there exists a unique local solution $\left\{x(t), t \in\left[t_{0}-\tau, \tau_{e}\right)\right\}$, where $\tau_{e}$ is the explosion time. Based on the definition of the initial condition $\eta$, we find that there exists an integer $r_{0}$ such that

$$
\|\eta\|=\sup _{\theta \in[-\tau, 0]}|\eta(\theta)|=\tau<r_{0} .
$$

For each integer $r \geq r_{0}$, define the stopping time

$$
\tau_{r}=T \wedge \inf \left\{t \in\left[t_{0}, \tau_{e}\right):\left|x^{n}(t)\right| \geq r\right\},
$$

and $\inf \emptyset=\infty$. Obviously, $\tau_{r}$ increases as $r \rightarrow \infty$. Define $\tau_{\infty}=\lim _{r \rightarrow \infty} \tau_{r}$, while $\tau_{\infty} \leq \tau_{e}$ a.s. Our goal is to prove that $\tau_{\infty}=T$ a.s., which implies that $\tau_{e}=T$, that is, the solution $\left\{x(t), t \in\left[t_{0}-\tau, T\right]\right\}$ does not explode in finite time.

Let us assume that $p \geq 2$. Using the Ito formula, we obtain

$$
\begin{aligned}
& E \sup _{s \in\left[t_{0}-\tau, t\right]}\left|x^{n}\left(s \wedge \tau_{r}\right)\right|^{p} \\
& \leq E\|\eta\|^{p}+p E \int_{t_{0}}^{t \wedge \tau_{r}}\left|x^{n}(s)\right|^{p-1}\left|A\left(s, x_{s}^{n}, z_{s}^{n}\right)\right| d s \\
&+\frac{p(p-1)}{2} E \int_{t_{0}}^{t \wedge \tau_{r}}\left|x^{n}(s)\right|^{p-2}\left|B\left(s, x_{s}^{n}, z_{s}^{n}\right)\right|^{2} d s \\
&+p E \sup _{s \in\left[t_{0}, t\right]}\left|\int_{t_{0}}^{s \wedge \tau_{r}}\left(x^{n}(u)\right)^{p-1} B\left(u, x_{u}^{n}, z_{u}^{n}\right) d w(u)\right| .
\end{aligned}
$$


Applying the Burkholder-Davis-Gundy inequality, we get

$$
\begin{aligned}
& p E \sup _{s \in\left[t_{0}, t\right]}\left|\int_{t_{0}}^{s \wedge \tau_{r}}\left(x^{n}(u)\right)^{p-1} B\left(u, x_{u}^{n}, z_{u}^{n}\right) d w(u)\right| \\
& \leq \sqrt{32} p E\left[\int_{t_{0}}^{t \wedge \tau_{r}}\left|x^{n}(s)\right|^{2 p-2}\left|B\left(s, x_{s}^{n}, z_{s}^{n}\right)\right|^{2} d s\right]^{\frac{1}{2}} \\
& \leq \sqrt{32} p E\left[\left(\sup _{s \in\left[t_{0}, t \wedge \tau_{r}\right]}\left|x^{n}(s)\right|^{p}\right)^{\frac{1}{2}}\left(\int_{t_{0}}^{t \wedge \tau_{r}}\left|x^{n}(s)\right|^{p-2}\left|B\left(s, x_{s}^{n}, z_{s}^{n}\right)\right|^{2} d s\right)^{\frac{1}{2}}\right] \\
& \leq \frac{1}{2} E \sup _{s \in\left[t_{0}-\tau, t\right]}\left|x^{n}\left(s \wedge \tau_{r}\right)\right|^{p}+16 p^{2} E \int_{t_{0}}^{t \wedge \tau_{r}}\left|x^{n}(s)\right|^{p-2}\left|B\left(s, x_{s}^{n}, z_{s}^{n}\right)\right|^{2} d s .
\end{aligned}
$$

Substituting (46) into (45), we find that

$$
\begin{aligned}
E \sup _{s \in\left[t_{0}-\tau, t\right]} \mid & \left.x^{n}\left(s \wedge \tau_{r}\right)\right|^{p} \\
\leq & 2 E\|\eta\|^{p}+2 p E \int_{t_{0}}^{t \wedge \tau_{r}}\left|x^{n}(s)\right|^{p-1}\left|A\left(s, x_{s}^{n}, z_{s}^{n}\right)\right| d s \\
& +\left[p(p-1)+32 p^{2}\right] E \int_{t_{0}}^{t \wedge \tau_{r}}\left|x^{n}(s)\right|^{p-2}\left|B\left(s, x_{s}^{n}, z_{s}^{n}\right)\right|^{2} d s .
\end{aligned}
$$

The application of the Young inequality yields

$$
\begin{aligned}
& \left|x^{n}(s)\right|^{p-1}\left|A\left(s, x_{s}^{n}, z_{s}^{n}\right)\right| \leq \frac{p-1}{p}\left|x^{n}(s)\right|^{p}+\frac{1}{p}\left|A\left(s, x_{s}^{n}, z_{s}^{n}\right)\right|^{p}, \\
& \left|x^{n}(s)\right|^{p-2}\left|B\left(s, x_{s}^{n}, z_{s}^{n}\right)\right|^{2} \leq \frac{p-2}{p}\left|x^{n}(s)\right|^{p}+\frac{2}{p}\left|B\left(s, x_{s}^{n}, z_{s}^{n}\right)\right|^{p} .
\end{aligned}
$$

On the basis of (48) and (49), the estimate (47) becomes

$$
\begin{aligned}
& E \sup _{s \in\left[t_{0}-\tau, t\right]}\left|x^{n}\left(s \wedge \tau_{r}\right)\right|^{p} \\
& \quad \leq 2 E\|\eta\|^{p}+3 p(11 p-21) \int_{t_{0}}^{t} E \sup _{u \in\left[t_{0}-\tau, s\right]}\left|x^{n}\left(u \wedge \tau_{r}\right)\right|^{p} d s \\
& \quad+2 E \int_{t_{0}}^{t \wedge \tau_{r}}\left|A\left(s, x_{s}^{n}, z_{s}^{n}\right)\right|^{p} d s+2(33 p-1) E \int_{t_{0}}^{t \wedge \tau_{r}}\left|B\left(s, x_{s}^{n}, z_{s}^{n}\right)\right|^{p} d s .
\end{aligned}
$$

Bearing in mind (42) and the fact that the Fresnel integral is bounded by the 
constant 0.977451424 (approximately 1), we have that

$$
\begin{aligned}
\left|A\left(s, x_{s}^{n}, z_{s}^{n}\right)\right|^{p} \leq & 4^{p-1}\left[\bar{\alpha}^{p}+\left(\frac{\bar{\beta}}{2}\right)^{p}\right]+4^{p-1} \bar{\alpha}^{p}\left|x^{n}(s)-z^{n}(s)\right|^{p} \\
& +4^{p-1} \bar{\beta}^{p}\left|\underline{h}\left(x_{s}^{n}\right)-\underline{h}\left(z_{s}^{n}\right)\right|^{p} .
\end{aligned}
$$

So, there exists $C_{1}>0$, such that

$$
C_{1}^{p} \geq 4^{p-1} \max \left\{\bar{\alpha}^{p}+\left(\frac{\bar{\beta}}{2}\right)^{p}, \bar{\beta}^{p}\right\}
$$

and

$$
\left|A\left(s, x_{s}^{n}, z_{s}^{n}\right)\right|^{p} \leq C_{1}^{p}\left[1+\left|x^{n}(s)-z^{n}(s)\right|^{p}+\left|\underline{h}\left(x_{s}^{n}\right)-\underline{h}\left(z_{s}^{n}\right)\right|^{p}\right] .
$$

In a view of (43), we observe that

$$
\left|B\left(s, x_{s}^{n}, z_{s}^{n}\right)\right|^{p} \leq 2^{p-1}\left[\bar{a}_{1}^{p}+\bar{a}_{1}^{p}\left|\underline{h}\left(x_{s}^{n}\right)-\underline{h}\left(z_{s}^{n}\right)\right|^{p}\right] .
$$

Thus, there exists $C_{2}>0$, such that $C_{2}^{p} \geq 2^{p-1} \bar{a}_{1}^{p}$ and

$$
\left|B\left(s, x_{s}^{n}, z_{s}^{n}\right)\right|^{p} \leq C_{2}^{p}\left[1+\left|\underline{h}\left(x_{s}^{n}\right)-\underline{h}\left(z_{s}^{n}\right)\right|^{p}\right] .
$$

Substituting (51) and (52) into (50), we have that, for all $t \in\left[t_{0}, T\right]$,

$$
\begin{aligned}
E \sup _{s \in\left[t_{0}-\tau, t\right]}\left|x^{n}\left(s \wedge \tau_{r}\right)\right|^{p} \leq & 2 E\|\eta\|^{p}+2\left[C_{1}^{p}+(33 p-1) C_{2}^{p}\right]\left(T-t_{0}\right) \\
& +3 p(11 p-21) \int_{t_{0}}^{t} E \sup _{u \in\left[t_{0}-\tau, s\right]}\left|x^{n}\left(u \wedge \tau_{r}\right)\right|^{p} d s \\
& +2 C_{1}^{p} E \int_{t_{0}}^{t \wedge \tau_{r}}\left|x^{n}(s)-z^{n}(s)\right|^{p} d s \\
& +2\left[C_{1}^{p}+(33 p-1) C_{2}^{p}\right] E \int_{t_{0}}^{t \wedge \tau_{r}}\left|\underline{h}\left(x_{s}^{n}\right)-\underline{h}\left(z_{s}^{n}\right)\right|^{p} d s .
\end{aligned}
$$

We will estimate the term

$$
\left|\underline{h}\left(x_{s}^{n}\right)-\underline{h}\left(z_{s}^{n}\right)\right|^{p}=\left|\underline{h}\left(x_{s}^{n}\right)-\underline{h}\left(x_{t_{k}}^{n}\right)\right|^{p}, s \in\left[t_{k}, t_{k+1} \wedge t\right], k \in\{0,1, \ldots, n-1\} .
$$

The application of the Hölder inequality yields

$$
\begin{aligned}
\left|\underline{h}\left(x_{s}^{n}\right)-\underline{h}\left(x_{t_{k}}^{n}\right)\right|^{p} & =\left|\int_{-\tau}^{0} \frac{1}{\tau}\left(x^{n}(s+\theta)-x^{n}\left(t_{k}+\theta\right)\right) d \theta\right|^{p} \\
& \leq \frac{1}{\tau} \int_{-\tau}^{0}\left|x^{n}(s+\theta)-x^{n}\left(t_{k}+\theta\right)\right|^{p} d \theta \\
& \leq 2^{p-1} \sup _{u \in\left[t_{0}-\tau, s\right]}\left|x^{n}(u)\right|^{p} .
\end{aligned}
$$


On the basis of (54), the expression (53) can be estimated as

$$
\begin{aligned}
E \sup _{s \in\left[t_{0}-\tau, t\right]} \mid x^{n}(s & \left.\wedge \tau_{r}\right)\left.\right|^{p} \\
& \leq H_{1}(p)+H_{2}(p) \int_{t_{0}}^{t} E \sup _{u \in\left[t_{0}-\tau, s\right]}\left|x^{n}\left(u \wedge \tau_{r}\right)\right|^{p} d s
\end{aligned}
$$

where

$$
\begin{aligned}
& H_{1}(p)=2 E\|\eta\|^{p}+2\left[C_{1}^{p}+(33 p-1) C_{2}^{p}\right]\left(T-t_{0}\right), \\
& H_{2}(p)=3 p(11 p-21)+2^{p+1} C_{1}^{p}+2^{p}(33 p-1) C_{2}^{p} .
\end{aligned}
$$

Then, the application of the Gronwall-Bellman lema yields

$$
E \sup _{s \in\left[t_{0}-\tau, t\right]}\left|x^{n}\left(s \wedge \tau_{r}\right)\right|^{p} \leq H_{1}(p) e^{H_{2}(p)\left(T-t_{0}\right)}, \quad r \geq r_{0}, t \in\left[t_{0}, T\right] .
$$

Particularly, we have that

$$
E \sup _{s \in\left[t_{0}-\tau, T\right]}\left|x^{n}\left(s \wedge \tau_{r}\right)\right|^{p} \leq H_{1}(p) e^{H_{2}(p)\left(T-t_{0}\right)}, \quad r \geq r_{0} .
$$

In a view of (44) and (56), we have that

$$
\begin{aligned}
r^{p} P\left\{\tau_{r} \leq T\right\} & \leq E\left[\sup _{s \in\left[t_{0}-\tau, t\right]}\left|x^{n}\left(s \wedge \tau_{r}\right)\right|^{p} I_{\left\{\tau_{r} \leq T\right\}}\right] \\
& \leq E \sup _{s \in\left[t_{0}-\tau, t\right]}\left|x^{n}\left(s \wedge \tau_{r}\right)\right|^{p} \\
& \leq H_{1}(p) e^{H_{2}(p)\left(T-t_{0}\right)}, \quad r \geq r_{0} .
\end{aligned}
$$

Thus, letting $r \rightarrow \infty$ in the last inequality we obtain that $P\left\{\tau_{\infty} \leq T\right\}=0$, that is $P\left\{\tau_{\infty}>T\right\}=1$. On the other hand, letting $r \rightarrow \infty$ in (56), we get

$$
E \sup _{s \in\left[t_{0}-\tau, T\right]}\left|x^{n}(s)\right|^{p} \leq H_{1}(p) e^{H_{2}(p)\left(T-t_{0}\right)} .
$$

So, we conclude that there exists a unique solution of Eq. (41) with bounded $p$-th moment, for any $p \geq 2$, which yields $\mathcal{A}_{6}$.

Theorem 2.1 gives the rate of the closeness in the $L^{p}$ sense, that is

$$
E \sup _{t \in\left[t_{0}-\tau, T\right]}\left|x(t)-x^{n}(t)\right|^{p} \leq H \delta_{n}^{p} .
$$


AN APPROXIMATE TAYLOR METHOD FOR STOCHASTIC FUNCTIONAL

\section{Conclusions}

In this paper, an analytic approximate method based on the application of the Taylor formula is considered for a class of SFDEs with coefficients satisfying polynomial conditions. The main results are the $L^{p}$ and a.s. convergence of the sequence of the approximate solutions to the exact solution of the initial equation. It should be emphasized that the rate of the $L^{p}$-convergence increases as the number of derivatives in the Taylor expansions of the coefficients of the initial equation increase. Besides the polynomial condition, the initial assumptions under which the main results are obtained include the existence, uniqueness and moment boundedness of both exact and approximate solutions. Through the example, all assumptions are verified by direct computation or using the existing results except the mentioned properties of the approximate solution, which are proved.

Acknowledgment: The research is supported by the Ministry of Education, Science and Technological Development of the Republic of Serbia, No. 451-03-9/2021-14/200124

\section{References}

[1] M. A. Atalla, Finite-difference approximations for stochastic differential equations, Probabilistic Methods for the Investigation of Systems with an Infinite number of Degrees of freedom, Inst. of Math. Acad. of Science USSR, Kiev, (1986) 11-16 (in Russian).

[2] M. A. Atalla, On one approximating method for stochastic differential equations, Asymptotic Methods for the Theory of Stochastic processes, Inst. of Math. Acad. of Science USSR, Kiev, (1987) 15-21 (in Russian).

[3] A. Bahar, X. Mao, Stochastic delay population dynamics, International J. Pure Appl. Math., 11 (2004) 377-400.

[4] L. Collatz, "Functional analysis and numerical mathematics", Academic Press, New York - San Francisco - London (1966).

[5] T. M. Flett, "Differential analysis", Cambridge University Press, Cambridge (1980).

[6] Y. Guo, W. Zhao, X. Ding, Input-to-state stability for stochastic multigroup models with multy-disperal and time-varying delay, App. Math. Comp., 343 (2019) 114-127.

[7] D. J. Higham, X. Mao, A. M. Stuart, Strong convergence of Euler-type methods for nonlinear stochastic differential equations, SIAM J. Numer. Anal., 40 (3) (2002) 1041-1063.

[8] M. Jansen, P. Pfaffelhuber, Stochastic gene expression with delay, J. Theor. Biol., 364 (2015) 355-363. 
[9] M. Jovanović, M. Krstić, The influence of time-dependent delay on behavior of stochastic population model with the Allee effect, App. Math. Modell., 39(2) (2015) 733-746.

[10] V.B. Kolmanovskii, V.R. Nosov, Stability of Functional Differential Equations, Academic Press, 1986.

[11] M. Krstić, The effect of stochastic perturbation on a nonlinear delay malaria epidemic model, Math. Comput. Simulat., 82(4) 558-569.

[12] M. Liu, C. Bai, Y. Jin, Population dynamical behavior of a two-predator one-prey stochastic model with time delay, Discrete Contin. Dyn. Sys. A, 37 (5) (2017) 2513-2538.

[13] Q. Liu, D. Jiang, T. Hayat, A. Alsaedi, Dynamics of a stochastic SIR epidemic model with distributed delay and degenerate diffusion, J. Frankl. Inst., 356 (13) (2019) 7347-7370.

[14] X. Mao, "Stochastic differential equations and applications", Horwood Publishing, Chichester, (2008).

[15] X. Mao, S. Sabanis, Numerical solutions of stochastic differential delay equations under local Lipschitz condition, J. Comput. Appl. Math., 151 (2003) 215-227.

[16] M. Milošević, M. Jovanović, S. Janković, An approximate method via Taylor series for stochastic functional differential equations, J. Math. Anal. Appl., 363 (2010) 128-137.

[17] M. Obradović, M. Milošević, Stability of a class of neutral stochastic differential equations with unbounded delay and Markovian switching and the Euler?Maruyama method, J. Comput. Appl. Math., 244-266 (2017).

[18] J. A. Oguntuase, On integral inequalities of Gronwall-Bellman-Bihari type in several variables, J. Ineq. Pure Appl. Math., 1 (2) (2000).

[19] B. Øksendal, A. Sulem, T. Zhang, Optimal control of stochastic delay equations and time-advanced backward stochastic differential equations, Adv. Appl. Probab., 43(2) (2011) 572-596.

[20] M. Song, L. Hu, X. Mao, L. Zhang, Khasminskii-type theorems for stochastic functional differential equations, Discrete and continuous dynamical systems, series B, 18 (6) (2013) 1697-1714.

[21] B. Tojtovska, S. Janković, General decay stability analysis of impulsive neural networks with mixed time delays, Neurocomputing, 142 (2014) 438-446.

[22] M. Vasilova, Asymptotic behavior of a stochastic Gilpin-Ayala predatorprey system with time-dependent delay, Math. Comp. Model., 57 (3-4) (2013) 764-781. 
[23] X. Wang, J. Yu, C. Li, H. Wang, T. Huang, J. Huang, Robust stability of stochastic fuzzy delayed neural networks with impulsive time window, Neural Networks, 67 (2015) 84-91.

[24] F. Wu, X. Mao, Numerical solutions of neutral stochastic functional differential equations, SIAM J. Numer. Anal. 46 (2008) 1821-1841.

Dušan D. Djordjević,

University of Niš,

Faculty of Sciences and Mathematics,

Višegradska 33, 18000 Niš, Serbia.

Email: djoledj91@gmail.com

Marija Milošević,

University of Niš,

Faculty of Sciences and Mathematics,

Višegradska 33, 18000 Niš, Serbia.

Email: 27marija.milosevic@gmail.com 